\title{
Simultaneous Sensing and Transmission for Cognitive Radios with Imperfect Signal Cancellation
}

\author{
Christos Politis, Sina Maleki, Christos Tsinos, Konstantinos Liolis, Symeon Chatzinotas, and Björn Ottersten
}

\begin{abstract}
In conventional cognitive radio systems, the secondary user employs a "listen-before-talk" paradigm, where it senses if the primary user is active or idle, before it decides to access the licensed spectrum. However, this method faces challenges with the most important being the reduction of the secondary user's throughput, as no data transmission takes place during the sensing period. In this context, the idea of simultaneous spectrum sensing and data transmission is proposed. The present work studies a system model where this concept is obtained through the collaboration of the secondary transmitter with the secondary receiver. First, the secondary receiver decodes the signal from the secondary transmitter, subsequently, removes it from the total received signal and then, carries out spectrum sensing in the remaining signal in order to decide about the presence/absence of the primary user. Different from the existing literature, this paper takes into account the imperfect signal cancellation, evaluating how the decoding errors affect the sensing reliability and derives the analytical expressions for the probability of false alarm. Finally, numerical results are presented illustrating the accuracy of the proposed analysis.
\end{abstract}

Index Terms-Cognitive radio, simultaneous sensing and transmission, Energy Detector, imperfect signal cancellation, truncated chi-squared distribution.

\section{INTRODUCTION}

$\mathbf{C}$ OGNITIVE radio has become a promising technology in wireless communications, because of its capability to be aware of the environment, and hence offering efficient use of the spectrum [1]-[2]. Spectrum sensing is the key functionality of a cognitive radio system that can be utilized in other applications as well, such as network management services [3] and interference detection [4]. Here, we are interested in using the information from spectrum sensing, for protecting the primary user (PU) from the interference caused by the secondary user (SU), or for allowing the SU to access the spectrum when it is not occupied by the PU. The common spectrum sensing techniques, in terms of the way that the cognitive radio users can detect the presence or absence of the PU are presented in [5], and include matched filter detection

Christos Politis, Sina Maleki, Christos Tsinos, Symeon Chatzinotas and Björn Ottersten are with SnT at the University of Luxembourg, Luxembourg. E-mails: christos.politis@uni.lu, sina.maleki@uni.lu, christos.tsinos@uni.lu, symeon.chatzinotas@uni.lu, and bjorn.ottersten@uni.lu. Konstantinos Liolis is with SES, Luxembourg. E-mail: Konstantinos.Liolis@ses.com.

This work is supported by Fond National de la Recherche Luxembourg (FNR), under the CORE projects Satellite Sensor Networks for Spectrum Monitoring (SATSENT) and Spectrum Management and Interference Mitigation in Cognitive Radio Satellite Networks (SeMiGod). A part of this work has been presented at the IEEE International Conference on Acoustics, Speech and Signal Processing (ICASSP) 2017.
[6], energy detection [7]-[10], cyclostationary detection [11] and eigenvalue based detection [12]-[14].

On the other hand, the spectrum sensing paradigms discussed in the literature, according to the way that the cognitive radio users can access the licensed spectrum, are divided into the following two categories: i) quiet [15] and ii) active [16]. In quiet spectrum sensing, the SU devotes $\tau$ units of time (quiet period) in order to sense the presence or absence of the PU user before it starts the transmission. If the frequency band is detected idle (the PU is absent), the SU employs the remaining frame duration $T-\tau$ for data transmission. This strategy is depicted in Figure 1a, where each frame is divided into two parts: i) the spectrum sensing period and ii) the data transmission period. The main advantage of this method is the hardware simplicity, as the switch from the sensing to communication mode can be obtained by using a single radio architecture [17]. However, this approach uses a quiet period for spectrum sensing resulting in the reduction of SU's throughput, as no data transmission takes place during the sensing period.

To address this issue, the idea of simultaneous sensing and data transmission has been proposed and the frame structure is presented in Figure 1b. These works are distinguished into two main types: i) techniques that apply the concept at the SU transmitter side [18]-[21] and ii) techniques that enable the cooperation between the SU transmitter and an inactive SU [16], [22] or between the SU transmitter and the SU receiver via a control channel [23]. These approaches are summarized as follows.

In the first category, the same CR device performs simultaneous sensing and communication, where the transmitter is equipped with both a sensing and transmit unit. The critical issue of this method is the self-interference, created between the sensing and communication path because of the close proximity of the antennas. Therefore, the functionality of this method is completely based on the ability to isolate the antennas of the transmit and sensing unit and cancel the selfinterference. In [18], an approach was proposed based on the idea of spatial filtering for achieving a tolerable level of isolation. However, a stronger isolation is required and is obtained by equipping the transmitter with redundant antennas. In [19], the work of [20] was extended by proposing a multiantenna structure, which adaptively uses spatial resources regarding the surrounding environment. However, this technique limits its applicability only to SUs equipped with multiple antennas. Furthermore, in [20] a "listen-and-talk" protocol was proposed that enables simultaneous sensing and transmission by adopting the Energy Detector (ED) as sensing scheme, where the threshold adaptively changes, in terms of the secondary 


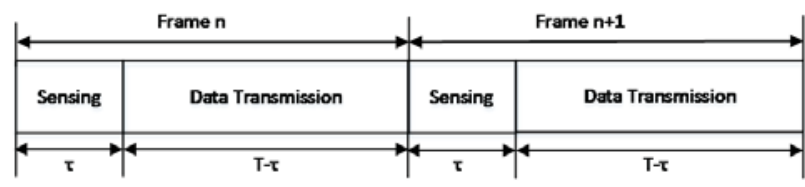

(a) Quiet spectrum sensing.

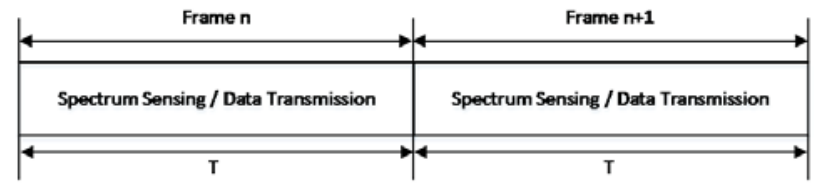

(b) Simultaneous spectrum sensing and data transmission.

Fig. 1: Frame structure.

transmitter activity. In [21] a two-phase concurrent sensing and transmission scheme was proposed employing a suitable control power mechanism. Nevertheless, an important drawback of these techniques is the requirement for extra dedicated hardware (antennas) for sensing that increases the cost of the system. Moreover, as mentioned earlier, these approaches introduce the phenomenon of self-interference, which degrades the sensing performance, and hence, self-interference cancellation schemes have to be adopted, which however, increase the power consumption of the system with their turn.

On the other hand, there are works which propose simultaneous sensing and transmission using an inactive SU. In [16] a cognitive radio system was proposed, which performs spectrum sensing through an inactive SU, while an active SU is transmitting. A similar analysis is proposed in [22], where a cognitive base station transmits data to some SUs using zero forcing, while some other SUs carry out spectrum sensing. However, again, these approaches face challenges, such as the extra power consumption and waste of resources by using an inactive SU or some other SUs for spectrum sensing.

In [23], a different concept was proposed, where the simultaneous spectrum sensing and data transmission is obtained through the collaboration of the SU transmitter and the SU receiver, which perform in different nodes. The SU transmitter is responsible for the data transmission, while the SU receiver decodes the signal from the secondary transmitter, removes it from the total received signal and carries out spectrum sensing in the remaining signal. The two main advantages of this technique compared to the approach at the transmitter's side are that i) it does not use extra antennas for the spectrum sensing, hence, it can be easily implemented in the current systems with no additional hardware change and also, ii) it does not face the problem of self-interference that described earlier. Furthermore, this approach offers much better detection performance than that of using inactive SUs, if we assume that the adopted detection scheme is the Energy Detector. The reason is that the decoding and cancellation of the SU transmitted signal is almost impossible by using the inactive SU, because the latter needs information about the channel, modulation and coding and etc., which are hardly available in practice. However, the work of [23] is under the ideal assumption of perfect signal decoding.

In this context, the contributions of this paper are three-fold:

- Unlike existing works that assume perfect signal cancellation, in this paper, we investigate simultaneous sensing and transmission taking the imperfect signal cancellation into account. Energy detection is then applied on the remaining signal to detect the presence or absence of the PU.
- We evaluate how the imperfect signal cancellation due to decoding errors, affects the sensing performance. In addition, we derive the sensing performance parameters i.e., probability of detection $\left(P_{D}\right)$ and probability of false alarm $\left(P_{F A}\right)$ for BPSK, QPSK, and general M-QAM SU signals.

- It is shown that the remaining signal, and consequently its energy follows a truncated distribution. Applying the concept of truncated distribution, we derive the mean and variance of a truncated central or non-central chisquared variable. Further, in combination with central limit theorem (CLT), the distribution, mean and variance of the sum of $N$ truncated central or non-central chisquared variables are derived. This is used to model the distribution of the energy detection test statistics. Finally, the approximated expressions are evaluated by numerical results which verify the accuracy.

The rest of the paper is organized as follows. In Section II, the system model and the proposed method are described. Section III presents the derivation of the $P_{F A}$ and $P_{D}$ for BPSK and QPSK SU modulated signals, while Section IV provides the expression of $P_{F A}$ for any M-QAM SU modulated scheme. Numerical results are illustrated in Section V. Finally, Section VI concludes the paper.

Notation: Bold-face lower case letters are used to declare vectors. $\mathcal{R}\{\cdot\}$ and $\mathcal{I}\{\cdot\}$ denote the real and the imaginary part of $\{\cdot\}$, respectively, while $E\{\cdot\}$ and $V\{\cdot\}$ represent the expectation and the variance of $\{\cdot\}$, respectively. The superscript $(\cdot)^{T}$ represents the transpose of $(\cdot)$. $\|\cdot\|$ denotes the standard vector norm, while $|\cdot|$ is the absolute value. The chi-squared distribution with $q$ degrees of freedom is denoted by $\chi_{q}^{2}$.

\section{SYSTEM MODEL}

\section{A. Signal Model}

We consider a cognitive radio system as shown in Figure 2, where the primary user transmitter (PU-Tx) and the secondary user transmitter/receiver (SU-Tx/Rx) are equipped with one antenna. The goal of this system is to detect if the PU is active or idle following the concept of simultaneous spectrum sensing and data transmission through the cooperation of the SU-Tx and the SU-Rx. Therefore, the detection problem can be formulated as the following binary hypothesis test, which is a baseband symbol sampled model:

$$
\begin{aligned}
& \mathcal{H}_{0}: \quad \mathbf{y}=h \mathbf{s}+\mathbf{w}, \\
& \mathcal{H}_{1}: \quad \mathbf{y}=\mathbf{x}_{p}+h \mathbf{s}+\mathbf{w},
\end{aligned}
$$

where $h$ denotes the scalar flat fading channel from the SUTx to the SU-Rx, which is assumed to be known at the 


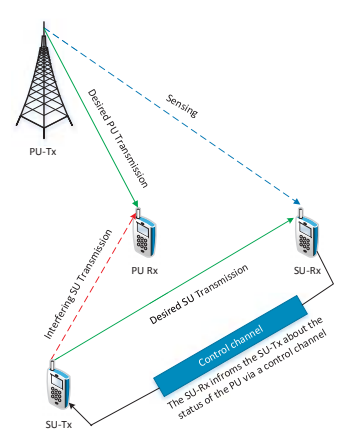

Fig. 2: System model.

secondary users as in [23], and it is also assumed to be real after the phase compensation with channel power $\gamma$, $\mathbf{s}=[s(1) \cdots s(N)]^{T}$ denotes an $N \times 1$ vector, which is the signal transmitted by the SU-Tx with power $P_{s}$ and it is a modulated signal, $\mathbf{x}_{p}=\left[x_{p}(1) \cdots x_{p}(N)\right]^{T}$ denotes an $N \times 1$ vector, referred to as the received (faded) signal from the PU-Tx, $\mathbf{w}=[w(1) \cdots w(N)]^{T}$ denotes an $N \times 1$ vector, which is the additive noise at the receiving antenna of the SU-Rx, modelled as an independent and identically distributed (i.i.d.) complex Gaussian vector with zero mean and covariance matrix given by $E\left\{\mathbf{w w}^{H}\right\}=\sigma_{w}^{2} \mathbf{I}_{N}$, where $\mathbf{I}_{N}$ denotes an identity matrix of size $N$, and $\mathbf{y}=[y(1) \cdots y(N)]^{T}$ denotes an $N \times 1$ vector, referred to as the total received signal at the SU-Rx.

As mentioned, in this paper we assume that the channel is known. However, in reality, the channel should be estimated. Therefore, the channel estimation error is an important factor which has to be taken into account in our analysis, constituting a valuable idea for future studies. Nevertheless, in Section V, we present preliminary results how this uncertainty affects the sensing performance of our proposed detector.

\section{B. Method Description}

In a cognitive radio network, the goal of the SU-Tx is to access the spectrum when it is not occupied by the PU and thus avoiding interference to the PU network (this is obtained by considering a high target probability of detection). Therefore, in the beginning, namely during the very first frame of cognitive data transmission, the SU-Tx divides the frame in two time slots: i) one sensing period ( $\tau$ units of time) and ii) one data transmission period ( $T$ units of time). If the PU is detected to be idle during the sensing period, the SU-Tx changes mode and starts the data transmission to the SU-Rx. Then, in the following frames the SU-Rx decodes the signal of the SU-Tx and removes it from the total received signal. Subsequently, a detector is applied to the remaining signal for spectrum sensing. At the end of the frame, if the SU detects a change in the state of the PU (e.g. the PU starts the transmission after the sensing period), then the SU-Rx informs the SU-Tx via a control channel and the latter stops the transmission in order to avoid causing interference to the PU. Hence, in the next frame, the SU-Tx, again, divides the frame in two time slots and the above process is repeated. However, at the end of the frame, if the SU detects that the frequency band is idle (absence of the

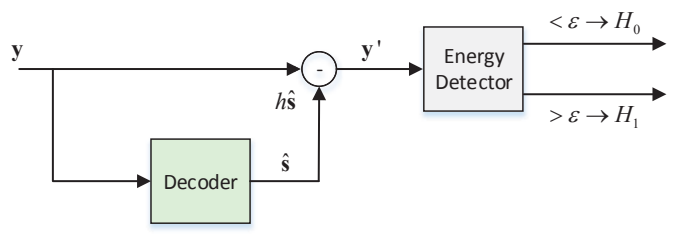

Fig. 3: Receiver structure of the proposed method for spectrum sensing and data transmission at the same time.

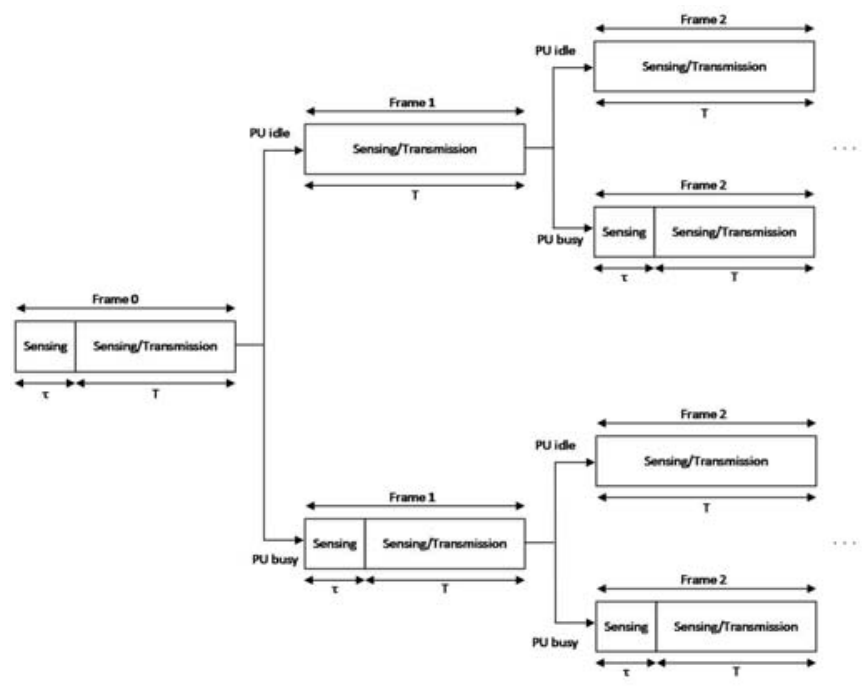

Fig. 4: Frame structure of the proposed simultaneous sensing and transmission method.

PU), there is no need to devote a period for sensing during the next frame, and then, the idea of simultaneous spectrum sensing and data transmission is applied improving the SU's throughput. The structure of the secondary receiver is depicted in Figure 3, while the frame structure of this method is presented in Figure 4.

\section{Proposed Algorithm}

The aforementioned methodology can be applied for any modulation scheme, but in this step, for simplicity, we consider that the transmitted signal from the SU is BPSK modulated and the noise is a real (not complex) Gaussian vector. Later, we will show how the proposed algorithm can be applied for QPSK and any M-QAM modulated signal with complex Gaussian noise. Hence, following these assumptions the detection problem of (1)-(2) can be reformulated via the following procedure.

1) After the initial stage of sensing, the $S U R x$ tries to decode the signal transmitted by the SU Tx, using the BPSK demodulator, which is based on the Euclidean distance [24]. Note that given that the transmitted symbol is $s=\sqrt{P_{s}}$, the correctly decoded signal is $\hat{s}=\sqrt{P_{s}}$, while the wrongly decoded signal is $\hat{s}=-\sqrt{P_{s}}$ or more generally

$$
\hat{s}=\left\{\begin{array}{lll}
+s & \rightarrow & \text { correct decoding } \\
-s & \rightarrow & \text { wrong decoding. }
\end{array}\right.
$$


2) Then, the decoded signal is removed from the total received signal and the new hypothesis test is expressed as follows:

$$
\begin{aligned}
\mathcal{H}_{0_{B}} & =\left\{\begin{array}{l}
\mathcal{H}_{00_{B}}: y^{\prime}(n)=w(n), \\
\mathcal{H}_{01_{B}}: y^{\prime}(n)=2 h s(n)+w(n),
\end{array}\right. \\
\mathcal{H}_{1_{B}} & =\left\{\begin{array}{l}
\mathcal{H}_{10_{B}}: y^{\prime}(n)=x_{p}(n)+w(n), \\
\mathcal{H}_{11_{B}}: y^{\prime}(n)=x_{p}(n)+2 h s(n)+w(n),
\end{array}\right.
\end{aligned}
$$

where $n=0,1, \ldots, N-1$, the index $B$ in the hypothesis test denotes the BPSK scenario, $\mathcal{H}_{00_{B}}$ and $\mathcal{H}_{10_{B}}$ correspond to the hypothesis of correct decoding of the received signal, while the PU is idle and active, respectively, while $\mathcal{H}_{01_{B}}$ and $\mathcal{H}_{11_{B}}$ represent the wrong decoding case, while the PU is idle and active, respectively. Now, it is clear that without considering the decoding errors (hypotheses $\mathcal{H}_{01_{B}}$ and $\left.\mathcal{H}_{11_{B}}\right)$, the new hypothesis test is the same as the one of quiet spectrum sensing, with the difference that here, the whole duration of the frame is used for spectrum sensing instead of a small quiet period.

3) The last step is the application of the ED in the remaining signal, examining how the decoding errors affect the sensing performance. We selected the ED as detection technique due to the fact that it does not require knowledge of the PU characteristics (modulation type, pulse shaping, etc.), which are often unknown. The ED is shown in (6)

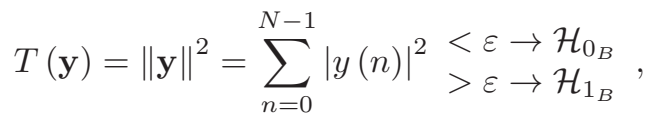

where $\varepsilon$ denotes a properly defined threshold, responsible for the decision about the presence or absence of the PU.

\section{Probability of false alarm and Probability of DETECTION}

The calculation of the detection threshold $\varepsilon$ is independent from the transmitted primary signal and hence, the evaluation of our proposed detection scheme is obtained via the derivation of the $P_{F A}$. Therefore, in this section, we mainly derive the $P_{F A}$, first for BPSK and then for QPSK SU modulated signals. Furthermore, for the same SU modulated signals, we derive the $P_{D}$ under the assumption that the primary user is Gaussiandistributed.

\section{A. Probability of false alarm for BPSK signals}

In this subsection, the probability of false alarm for the BPSK case $\left(P_{F A_{B}}\right)$ is determined by Theorem 1, which is subsequently proved.

Theorem 1: Consider a secondary user with one receive antenna, which collects a large number of samples $N$. The SU-Rx decodes the received samples, removes them from the total received signal and applies an ED in the remaining signal. Then, the $P_{F A_{B}}$ is defined by

$$
P_{F A_{B}}=\sum_{k=0}^{N}\left(\begin{array}{c}
N \\
k
\end{array}\right) P_{k_{B}} P_{e_{B}}^{k}\left(1-P_{e_{B}}\right)^{N-k},
$$

where $k$ denotes the number of wrong decoded bits, $P_{\mathrm{e}_{\mathrm{B}}}=$ $Q\left(\sqrt{\frac{\gamma P_{s}}{\sigma_{w}^{2}}}\right)$ is the probability of bit error for BPSK [24] and
$P_{k_{B}}$ is the probability of false alarm for the case that $k$ bits are decoded wrongly, which can be approximated as follows:

$$
P_{k_{B}}=Q\left(\frac{\varepsilon-\mu_{\mathcal{H}_{0_{B}}}}{\sqrt{V_{\mathcal{H}_{0_{B}}}}}\right),
$$

where $\mu_{\mathcal{H}_{0_{B}}}$ and $V_{\mathcal{H}_{0_{B}}}$ are the mean and variance of the test statistic $T\left(\mathbf{y}^{\prime} \mid \mathcal{H}_{0_{B}}\right)$, respectively.

Proof: The Binomial distribution [25] is used for the proof of the first part of Theorem 1, due to the fact that considering independent experiments, the number of wrong decoded bits is different in each of them, ranging from 0 to $N$ with a corresponding probability. For the second part, the probability of false alarm $P_{k_{B}}$ of the ED of (6) is determined by $P_{k_{B}}(\varepsilon)=$ $\operatorname{Pr}\left(T\left(\mathbf{y}^{\prime}\right)>\varepsilon \mid \mathcal{H}_{0_{B}}\right)$, where the derivation of the distribution of the test statistic $T\left(\mathbf{y}^{\prime} \mid \mathcal{H}_{0_{B}}\right)$ is required.

Focusing on $\mathcal{H}_{00_{B}}$, we can notice that the remaining signal, after the cancellation of the correct decoded signal, consists only of noise. This vector includes the set of noise values, which let the BPSK demodulator to decide correctly about the transmitted symbol. Then, the Cumulative Distribution Function (CDF) of the remaining signal under $\mathcal{H}_{00_{B}}$ can be written as follows:

$$
\begin{aligned}
F_{y}\left(g \mid \mathcal{H}_{00_{B}}\right) & =P\left\{y \leq g \mid \mathcal{H}_{00_{B}}\right\} \\
& =P\left\{y \leq g \mid c_{B}, s=+\sqrt{P_{s}}\right\} P\left(s=+\sqrt{P_{s}}\right) \\
& +P\left\{y \leq g \mid c_{B}, s=-\sqrt{P_{s}}\right\} P\left(s=-\sqrt{P_{s}}\right) \\
& =\frac{1}{2} P\left\{w \leq g \mid g \geq-\sqrt{P_{s}}\right\} \\
& +\frac{1}{2} P\left\{w \leq g \mid g \leq+\sqrt{P_{s}}\right\},
\end{aligned}
$$

where the symbols are assumed to be of equal probability $\left(P\left(s=+\sqrt{P_{s}}\right)=P\left(s=-\sqrt{P_{s}}\right)=1 / 2\right), c_{B}$ denotes the correct decision event under the BPSK scenario and $F_{y}\left(g \mid \mathcal{H}_{00_{B}}\right)$ represents the CDF of $y$ under $\mathcal{H}_{00_{B}}$. As it can be seen, the CDF is related to two cases: i) correct decoding given that the transmitted symbol is $s=+\sqrt{P_{s}}$ or ii) correct decoding given that the transmitted symbol is $s=-\sqrt{P_{s}}$. Then, the distribution of noise for both scenarios is depicted in Figures 5a and 5b. A very interesting information which can be extracted by these two figures is that the received signal is always decoded correctly in the region where $-\sqrt{\frac{\gamma P_{s}}{\sigma_{w}^{2}}} \leq w \leq+\sqrt{\frac{\gamma P_{s}}{\sigma_{w}^{2}}}$, while it is sometimes decoded correctly and some other wrongly in the region where $w \geq \sqrt{\frac{\gamma P_{s}}{\sigma_{w}^{2}}}$ or $w \leq-\sqrt{\frac{\gamma P_{s}}{\sigma_{w}^{2}}}$, based on the fact if the transmitted symbol is $s=\sqrt{P_{s}}$ or $s=-\sqrt{P_{s}}$. This analysis shows that the remaining noise under $\mathcal{H}_{00_{B}}$ follows a truncated normal distribution [26]-[27] in the following intervals: i)

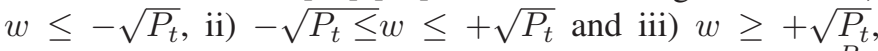
where for the rest of this paper, we assume that $P_{t}=\frac{\gamma P_{s}}{\sigma_{w}^{2}}$. Now, it becomes clear that we should determine what is the distribution of the sum of $N$ truncated central and/or noncentral chi-squared random variables. However, the closed form expression of this distribution is not mathematically tractable. Therefore, we should examine if the distribution of the test statistic $T\left(\mathbf{y}^{\prime} \mid \mathcal{H}_{0_{B}}\right)$ can be approximated by using the CLT 


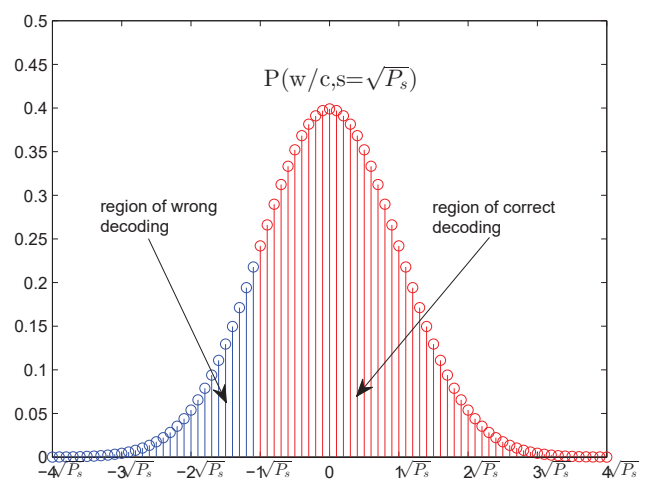

(a) Correct decoding of $s=\sqrt{P_{s}}=1$.

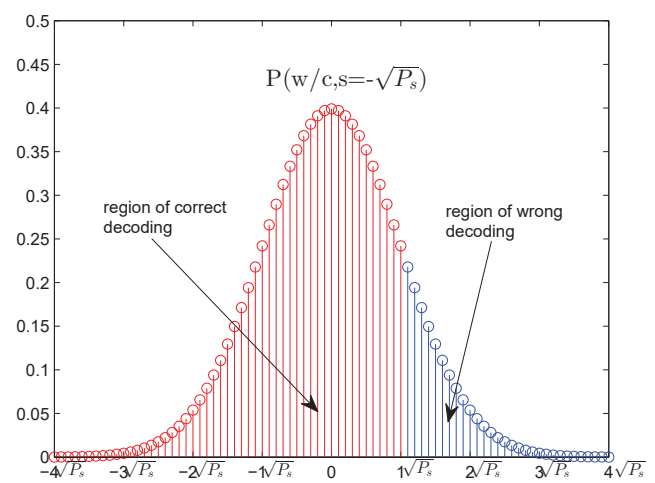

(b) Correct decoding of $s=-\sqrt{P_{s}}=-1$.

Fig. 5: Distribution of noise under the hypothesis $\mathcal{H}_{00_{B}}$.

[28].

The test statistic $T\left(\mathbf{y}^{\prime} \mid \mathcal{H}_{0_{B}}\right)$ consists of variables under one of the following cases: i) always correct decoding, ii) always wrong decoding and iii) sometimes correct and some other wrong decoding. The first two categories include a sequence of i.i.d random variables and hence, assuming a large number of samples the CLT can be applied. However, an independent but not identically distributed sequence is involved in the third case. Nevertheless, even in this case, the CLT can be used since the Lyapunov's and Lindeberg's conditions for non-identical variables [29] are satisfied. Thus, the mean and variance of $T\left(\mathbf{y}^{\prime} \mid \mathcal{H}_{0_{B}}\right)$ are respectively given by $\mu_{\mathcal{H}_{0 B}}=(N-k) \mu_{\mathcal{H}_{00 B}}+$ $k \mu_{\mathcal{H}_{01 B}}$ and $V_{\mathcal{H}_{0 B}}=(N-k) V_{\mathcal{H}_{00 B}}+k V_{\mathcal{H}_{01 B}}$, where $\mu_{\mathcal{H}_{00} B}$, $\mu_{\mathcal{H}_{01} B}, V_{\mathcal{H}_{00} B}$ and $V_{\mathcal{H}_{01} B}$ are the mean and variance of the test statistic $T\left(y^{\prime} \mid \mathcal{H}_{00_{B}}\right)$ and $T\left(y^{\prime} \mid \mathcal{H}_{01_{B}}\right)$, respectively, with $y^{\prime}$ meaning only one sample.

Hence, the derivation of the mean and variance for $T\left(y^{\prime} \mid \mathcal{H}_{00_{B}}\right)$ and $T\left(y^{\prime} \mid \mathcal{H}_{01_{B}}\right)$ is required. However, the calculation of these parameters is obtained with the help of the following three lemmas. Lemma 1 is valid for all truncated central chi-squared variables, Lemma 2 is valid, only when the non-central chi-squared variable is truncated to the interval $[a, b]$ where $0 \leq a \leq b<\infty$, while when $b=\infty$, Lemma 3 is used to evaluate the truncated non-central chi-squared variable.

Lemma 1: The mean $\mu_{c}^{[a, b]}$ and variance $V_{c}^{[a, b]}$ of a central chi-squared variable with one degree of freedom, truncated to the interval $[a, b]$ where $0 \leq a \leq b \leq \infty$, is given by

$$
\begin{gathered}
\mu_{c}^{[a, b]}=1+2\left[\frac{a f_{\chi_{1}^{2}}(a)-b f_{\chi_{1}^{2}}(b)}{F_{\chi_{1}^{2}}(b)-F_{\chi_{1}^{2}}(a)}\right], \\
V_{c}^{[a, b]}=2-4\left[\frac{a f_{\chi_{1}^{2}}(a)-b f_{\chi_{1}^{2}}(b)}{F_{\chi_{1}^{2}}(b)-F_{\chi_{1}^{2}}(a)}\right]^{2} \\
+2\left[\frac{a^{2} f_{\chi_{1}^{2}}(a)+a f_{\chi_{1}^{2}}(a)-b^{2} f_{\chi_{1}^{2}}(b)-b f_{\chi_{1}^{2}}(b)}{F_{\chi_{1}^{2}}(b)-F_{\chi_{1}^{2}}(a)}\right],
\end{gathered}
$$

where $F_{\chi_{1}^{2}}$ and $f_{\chi_{1}^{2}}$ denote respectively the CDF and the Probability Density Function (PDF) of a central chi-squared variable with one degree of freedom.

Proof: Let $F_{\chi_{1 T}^{2}}^{[a, b]}$ and $f_{\chi_{1 T}^{2}}^{[a, b]}$ denote respectively the CDF and PDF of a central chi-squared variable with one degree of freedom, truncated to the interval $[a, b]$ where $0 \leq a \leq b \leq \infty$. For the untracated case, i.e. $a=0$ and $b=\infty$, we drop the superscript and note only $F_{\chi_{1}^{2}}$ and $f_{\chi_{1}^{2}}$. Then, for $x \in[a, b]$, $F_{\chi_{1 T}^{2}}^{[a, b]}(x)=\frac{F_{\chi_{1}^{2}}(x)-F_{\chi_{1}^{2}}(a)}{F_{\chi_{1}^{2}}(b)-F_{\chi_{1}^{2}}(a)}$ and $f_{\chi_{1 T}^{2}}^{[a, b]}(x)=\frac{f_{\chi_{1}^{2}}(x)}{F_{\chi_{1}^{2}}(b)-F_{\chi_{1}^{2}}(a)}$.

According to [30], the moment generating function (MGF) for a truncated central chi-squared random variable $X \sim \chi_{1 T}^{2}$ is given as follows:

$M_{X}(t)=\left[\frac{F_{\chi_{1}^{2}}(b(1-2 t))-F_{\chi_{1}^{2}}(a(1-2 t))}{F_{\chi_{1}^{2}}(b)-F_{\chi_{1}^{2}}(a)}\right](1-2 t)^{-1 / 2}$.

Then, we can use the MGF to calculate the mean and variance of a truncated central chi-squared variable with one degree of freedom as follows: $\mu_{c}^{[a, b]}=E\{X \mid a \leq X \leq b\}=\left.M_{X}^{\prime}(t)\right|_{t=0} \quad$ and $V_{c}^{[a, b]}=V\{X \mid a \leq X \leq b\}=E\left\{X^{2} \mid a \leq X \leq b\right\}-$ $(E\{X \mid a \leq X \leq b\})^{2}$, where

$$
\begin{aligned}
& E\left\{X^{2} \mid a \leq X \leq b\right\}=\left.M_{X}^{\prime \prime}(t)\right|_{t=0} \\
& =3+4\left[\frac{a f_{\chi_{1}^{2}}(a)-b f_{\chi_{1}^{2}}(b)}{F_{\chi_{1}^{2}}(b)-F_{\chi_{1}^{2}}(a)}\right] \\
& +2\left[\frac{a^{2} f_{\chi_{1}^{2}}(a)+a f_{\chi_{1}^{2}}(a)-b^{2} f_{\chi_{1}^{2}}(b)-b f_{\chi_{1}^{2}}(b)}{F_{\chi_{1}^{2}}(b)-F_{\chi_{1}^{2}}(a)}\right] .
\end{aligned}
$$

Lemma 2: The mean $\mu_{c}^{[a, b]}$ and variance $V_{c}^{[a, b]}$ of a noncentral chi-squared variable with one degree of freedom and non-centrality parameter $\lambda$, truncated to the interval $[a, b]$ where $0 \leq a \leq b<\infty$, is given by

$$
\mu_{n c}^{[a, b]}=\frac{F_{\chi_{3, \lambda}^{2}}(b)-F_{\chi_{3, \lambda}^{2}}(a)+\lambda\left[F_{\chi_{5, \lambda}^{2}}(b)-F_{\chi_{5, \lambda}^{2}}(a)\right]}{F_{\chi_{1, \lambda}^{2}}(b)-F_{\chi_{1, \lambda}^{2}}(a)},
$$




$$
\begin{aligned}
V_{n c}^{[a, b]} & =\frac{3\left[F_{\chi_{5, \lambda}^{2}}(b)-F_{\chi_{5, \lambda}^{2}}(a)\right]}{F_{\chi_{1, \lambda}^{2}}(b)-F_{\chi_{1, \lambda}^{2}}(a)} \\
& +\frac{6 \lambda\left[F_{\chi_{7, \lambda}^{2}}(b)-F_{\chi_{7, \lambda}^{2}}(a)\right]}{F_{\chi_{1, \lambda}^{2}}(b)-F_{\chi_{1, \lambda}^{2}}(a)} \\
& +\frac{\lambda^{2}\left[F_{\chi_{9, \lambda}^{2}}(b)-F_{\chi_{9, \lambda}^{2}}(a)\right]}{F_{\chi_{1, \lambda}^{2}}(b)-F_{\chi_{1, \lambda}^{2}}(a)}-\left(\mu_{n c}^{[a, b]}\right)^{2},
\end{aligned}
$$

where $F_{\chi_{1, \lambda}^{2}}$ denotes the CDF of a non-central chi-squared variable with one degree of freedom and non-centrality parameter $\lambda$.

Proof: Let $F_{\chi_{1 T, \lambda}^{2}}^{[a, b]}$ denotes the CDF of a non-central chisquared variable with one degree of freedom, truncated to the interval $[a, b]$ where $0 \leq a \leq b<\infty$ and non-centrality parameter $\lambda$. For the non-truncated case, i.e. $a=0$ and $b=$ $\infty$, we drop the superscript and note only $F_{\chi_{1, \lambda}^{2}}$. Then, for $y \in[a, b], F_{\chi_{1 T, \lambda}^{2}}^{[a, b]}(y)=\frac{F_{\chi_{1, \lambda}^{2}}(y)-F_{\chi_{1, \lambda}^{2}}(a)}{F_{\chi_{1, \lambda}^{2}}^{2}(b)-F_{\chi_{1, \lambda}^{2}}^{2}(a)}$.

According to [31], the moments of the truncated non-central chi-squared distribution can be calculated as follows

$$
E\left\{T^{i}\right\}=\frac{h(i, p, \lambda)}{h(0, p, \lambda)},
$$

where $i \in\{0,1,2, \ldots\}, h(0, p, \lambda)=F_{\chi_{p, \lambda}^{2}}(b)-F_{\chi_{p, \lambda}^{2}}(a)$ and

$$
\begin{aligned}
h(i, p, \lambda) & =2^{i} \sum_{k=0}^{i}\left(\begin{array}{c}
i \\
k
\end{array}\right)\left(\frac{\lambda}{2}\right)^{k} \frac{\Gamma\left(\frac{p}{2}+i\right)}{\Gamma\left(\frac{p}{2}+k\right)} \times \\
& \times h(0, p+2 i+2 k, \lambda) .
\end{aligned}
$$

Then, the mean and variance of a truncated non-central chisquared variable $Y$ with one degree of freedom is derived as follows $\mu_{n c}^{[a, b]}=E\{Y \mid a \leq Y \leq b\}=E\{Y \mid a \leq Y \leq b\}$ and $V_{n c}^{[a, b]}=V\{Y \mid a \leq Y \leq b\}=E\left\{Y^{2} \mid a \leq Y \leq b\right\}-$ $(E\{Y \mid a \leq Y \leq b\})^{2}$.

Lemma 3: The mean $\mu_{n c \text { inf }}^{[-\infty, a]}$ and $V_{n c \text { inf }}^{[-\infty, a]}$ of a squared Gaussian variable, where the Gaussian variable is truncated to the interval $[a, \infty]$ or $[-\infty, \alpha]$, with mean $\mu$ and variance $\sigma^{2}$, is given by

$$
\begin{aligned}
\mu_{n c \text { inf }}^{[-\infty, a]} & =\mu^{2}-2 \mu \sigma \frac{f_{x}(d)}{F_{x}(d)}+\sigma^{2}\left(1-d \frac{f_{x}(d)}{F_{x}(d)}\right), \\
V_{n c \text { inf }}^{[-\infty, a]} & =\mu^{4}-4 \mu^{3} \sigma \frac{f_{x}(d)}{F_{x}(d)}+6 \mu^{2} \sigma^{2}\left(1-d \frac{f_{x}(d)}{F_{x}(d)}\right) \\
& +4 \mu \sigma^{3}\left(-d^{2} \frac{f_{x}(d)}{F_{x}(d)}-2 \frac{f_{x}(d)}{F_{x}(d)}\right) \\
& +\sigma^{4}\left(-d^{3} \frac{f_{x}(d)}{F_{x}(d)}-3 d \frac{f_{x}(d)}{F_{x}(d)}+3\right)-\left(\mu_{n c \text { inf }}^{[-\infty, a]}\right)^{2},
\end{aligned}
$$

where $d=a-\mu$ and $F_{x}, f_{x}$ denote the CDF and DPF of a normally distributed variable $x$.

Proof: Let $x=\mu+w$ be a normally distributed random variable with mean $\mu$ and variance $\sigma^{2}$, truncated to the interval $[-\infty, a]$. Then, the mean of the truncated variable $x^{2}$ is given by

$$
\begin{aligned}
\mu_{n c \text { inf }}^{[-\infty, a]} & =E\left\{x^{2} \mid x \leq a\right\} \\
& =E\left\{x^{2} \mid x \leq a\right\}=E\left\{|\mu+w|^{2} \mid \mu+w \leq a\right\} \\
& =\mu^{2}+2 \mu E\{w \mid w \leq a-\mu\}+E\left\{w^{2} \mid w \leq a-\mu\right\},
\end{aligned}
$$

while the variance is given by

$$
\begin{aligned}
& V_{n c \text { inf }}^{[-\infty, a]}=V\left\{x^{2} \mid x \leq a\right\} \\
& =E\left\{x^{4} \mid x \leq a\right\}-\left(E\left\{x^{2} \mid x \leq a\right\}\right)^{2} \\
& =E\left\{|\mu+w|^{4} \mid \mu+w \leq a\right\}-\left(E\left\{|\mu+w|^{2} \mid \mu+w \leq a\right\}\right)^{2} \\
& =\mu^{4}+4 \mu^{3} E\{w \mid w \leq a-\mu\}+6 \mu^{2} E\left\{w^{2} \mid w \leq a-\mu\right\} \\
& +4 \mu E\left\{w^{3} \mid w \leq a-\mu\right\}+E\left\{w^{4} \mid w \leq a-\mu\right\} \\
& -\left(\mu_{n c \text { inf }}^{[-\infty, a]}\right)^{2}
\end{aligned}
$$

Then, according to [32], the expression of $E\left\{u^{m} \mid u \leq a\right\}$, for some fixed $a$ is given by

$$
E\left\{u^{m} \mid u \leq a\right\}=\sum_{r=0}^{m}\left(\begin{array}{c}
m \\
r
\end{array}\right) \mu^{m-r} \sigma^{r} I_{r},
$$

where

$$
I_{r}=-d^{r-1} \frac{f_{x}(d)}{F_{x}(d)}+(r-1) I_{r-2},
$$

with the following initial conditions: i) $I_{0}=1$ and ii) $I_{1}=-\frac{f_{x}(d)}{F_{x}(d)}$. Therefore, using (21) and (22) and the fact that the noise has been assumed to be Gaussian with zero mean, the first moments of the truncated distribution are derived as follows: $E\left\{w^{0} \mid w \leq a\right\}=I_{0}=1, E\left\{w^{1} \mid w \leq a\right\}=$ $\sigma I_{1}=-\sigma \frac{f_{x}(d)}{F_{x}(d)}, E\left\{w^{2} \mid w \leq a\right\}=\sigma^{2} I_{2}=\sigma^{2}\left(1-d \frac{f_{x}(d)}{F_{x}(d)}\right)$, $E\left\{w^{3} \mid w \leq a\right\}=\sigma^{3} I_{3}=\sigma^{3}\left(-d^{2} \frac{f_{x}(d)}{F_{x}(d)}-2 \frac{f_{x}(d)}{F_{x}(d)}\right)$ and $E\left\{w^{4} \mid w \leq a\right\}=\sigma^{4} I_{4}=\sigma^{4}\left(-d^{3} \frac{f_{x}(d)}{F_{x}(d)}-3 d \frac{f_{x}(d)}{F_{x}(d)}+3\right)$. Finally, using (19)-(22), we can prove the mean and variance of (17) and (18), respectively.

Then, with the results of Lemma 1, we can develop Theorem 2, which defines the mean $\mu_{\mathcal{H}_{00}}$ and variance $V_{\mathcal{H}_{00}}$, required for the calculation of $\mu_{\mathcal{H}_{0_{B}}}$ and $V_{\mathcal{H}_{0_{B}}}$.

Theorem 2: Consider a secondary user with one receive antenna, which collects a number of samples $N$. The SU-Rx decodes the samples, removes them from the total received signal and applies the Energy Detector in the remaining signal. Then, the mean and variance of $T\left(y^{\prime} \mid \mathcal{H}_{00_{B}}\right)$ can be respectively defined as follows:

$$
\begin{aligned}
\mu_{\mathcal{H}_{00_{B}}} & =\mu_{c}^{\left[0, P_{t}\right]} P_{w}^{\left[-\sqrt{P_{t}}, \sqrt{P_{t}}\right]}+\mu_{c}^{\left[P_{t}, \infty\right]} P_{w}^{\left[\sqrt{P_{t}}, \infty\right]} \\
V_{\mathcal{H}_{00_{B}}} & =V_{c}^{\left[0, P_{t}\right]} P_{w}^{\left[-\sqrt{P_{t}}, \sqrt{P_{t}}\right]}+V_{c}^{\left[P_{t}, \infty\right]} P_{w}^{\left[\sqrt{P_{t}}, \infty\right]} \\
& +\left(\mu_{c}^{\left[P_{t}, \infty\right]}\right)^{2}\left(1-P_{w}^{\left[\sqrt{P_{t}}, \infty\right]}\right) P_{w}^{\left[\sqrt{P_{t}}, \infty\right]} \\
& +\left(\mu_{c}^{\left[0, P_{t}\right]}\right)^{2}\left(1-P_{w}^{\left[-\sqrt{P_{t}}, \sqrt{P_{t}}\right]}\right) P_{w}^{\left[-\sqrt{P_{t}}, \sqrt{P_{t}}\right]} \\
& -2 \mu_{c}^{\left[0, P_{t}\right]} \mu_{c}^{\left[P_{t}, \infty\right]} P_{w}^{\left[-\sqrt{P_{t}}, \sqrt{P_{t}}\right]} P_{w}^{\left[\sqrt{P_{t}}, \infty\right]},
\end{aligned}
$$


where $\mu_{c}^{\left[0, P_{t}\right]}, V_{c}^{\left[0, P_{t}\right]}, \mu_{c}^{\left[P_{t}, \infty\right]}$ and $V_{c}^{\left[P_{t}, \infty\right]}$ are defined by Lemma 1 , while $P_{w}^{\left[-\sqrt{P_{t}}, \sqrt{P_{t}}\right]}$ and $P_{w}^{\left[\sqrt{P_{t}}, \infty\right]}$ are expressed as follows: $P_{w}^{\left[-\sqrt{P_{t}}, \sqrt{P_{t}}\right]}=\frac{P\left(-\sqrt{P_{t}} \leq w \leq \sqrt{P_{t}}\right)}{P_{\mathrm{c}_{\mathrm{B}}}}=$ $\frac{F_{y}\left(\sqrt{P_{t}}\right)-F_{y}\left(-\sqrt{P_{t}}\right)}{P_{\mathrm{c}_{\mathrm{B}}}}$ and $P_{w}^{\left[\sqrt{P_{t}}, \infty\right]}=\frac{P\left(w \geq \sqrt{P_{t}}\right)}{P_{\mathrm{c}_{\mathrm{B}}}}=\frac{F_{y}\left(-\sqrt{P_{t}}\right)}{P_{\mathrm{c}_{\mathrm{B}}}}$, where $F_{y}(\cdot)$ is the $\mathrm{CDF}$ of a normally distributed variable $y$ and $P_{\mathrm{C}_{\mathrm{B}}}$ is the probability of correct decision for BPSK [33].

Proof: The proof of (23) and (24) is described in Appendix $\mathrm{A}$ and $\mathrm{B}$, respectively.

In a similar manner, following the results of Lemma 2 and 3 , we develop Theorem 3, which defines the mean $\mu_{\mathcal{H}_{01_{B}}}$ and variance $V_{\mathcal{H}_{01_{B}}}$, required for the calculation of $\mu_{\mathcal{H}_{0_{B}}}$ and $V_{\mathcal{H}_{0_{B}}}$.

Theorem 3: Consider a secondary user with one receive antenna, which collects a number of samples $N$. The SU-Rx decodes the samples, removes them from the total received signal and applies the Energy Detector in the remaining signal. Then, the mean and variance of $T\left(y^{\prime} \mid \mathcal{H}_{01_{B}}\right)$ can be respectively defined as follows:

$$
\begin{aligned}
\mu_{\mathcal{H}_{01_{B}}} & =\mu_{n c}^{\left[0, P_{t}\right]} P_{w}^{\left[\sqrt{P_{t}}, 3 \sqrt{P_{t}}\right]}+\mu_{n c i n f}^{\left[3 \sqrt{P_{t}}, \infty\right]} P_{w}^{\left[3 \sqrt{P_{t}}, \infty\right]}, \\
V_{\mathcal{H}_{01_{B}}} & =V_{n c}^{\left[0, P_{t}\right]} P_{w}^{\left[\sqrt{P_{t}}, 3 \sqrt{P_{t}}\right]}+V_{n c i n f}^{\left[3 \sqrt{P_{t}}, \infty\right]} P_{w}^{\left[3 \sqrt{P_{t}}, \infty\right]} \\
& +\left(\mu_{n c i n f}^{\left[3 \sqrt{P_{t}}, \infty\right]}\right)^{2}\left(1-P_{w}^{\left[3 \sqrt{P_{t}}, \infty\right]}\right) P_{w}^{\left[3 \sqrt{P_{t}}, \infty\right]} \\
& \left.+\left(\mu_{n c}^{\left[0, P_{t}\right]}\right)^{2}\left(1-P_{w}^{\left[\sqrt{P_{t}}, 3 \sqrt{P_{t}}\right]}\right) P_{w}^{\left[\sqrt{P_{t}}, 3 \sqrt{P_{t}}\right]}\right] \\
& -2 \mu_{n c}^{\left[0, P_{t}\right]} \mu_{n c i n f}^{\left[3 \sqrt{P_{t}}, \infty\right]} P_{w}^{\left[\sqrt{P_{t}}, 3 \sqrt{P_{t}}\right]} P_{w}^{\left[3 \sqrt{P_{t}}, \infty\right]},
\end{aligned}
$$

where $P_{w}^{\left[\sqrt{P_{t}}, 3 \sqrt{P_{t}}\right]}=\frac{P\left(\sqrt{P_{t}} \leq w \leq 3 \sqrt{P_{t}}\right)}{P_{c_{B} B}}=\frac{F_{x}\left(3 \sqrt{P_{t}}\right)-F_{x}\left(\sqrt{P_{t}}\right)}{P_{c_{-} \mathrm{B}}}$, $P_{w}^{\left[3 \sqrt{P_{t}}, \infty\right]}=\frac{P\left(w \geq 3 \sqrt{P_{t}}\right)}{P_{c_{-} \mathrm{B}}}=\frac{\bar{F}_{x}\left(-3 \sqrt{P_{t}}\right)}{P_{c_{-} \mathrm{B}}}$ and $\mu_{n c}^{\left[0, P_{t}\right]}, V_{n c}^{\left[0, P_{t}\right]}$, $\mu_{\text {ncinf }}^{\left[P_{t}, \infty\right]}, V_{n c i n f}^{\left[P_{t}, \infty\right]}$ are defined by Lemma 2 and Lemma 3.

Proof: The proof of (25) and (26) is similar to that presented in Appendix A and B.

Therefore, all the parameters of (7) have been derived. However, as mentioned, the required condition for the evaluation of the detector is the proper calculation of the detection threshold $\varepsilon$, which is significantly complex through (7), particularly as $N$ increases. For this reason, an approximated expression for the probability of false alarm is derived in this paper as follows:

$$
P_{F A_{B_{a p r}}}=Q\left(\frac{\varepsilon-N\left(1-P_{e_{B}}\right) \mu_{\mathcal{H}_{00_{B}}}-N P_{e_{B}} \mu_{\mathcal{H}_{01_{B}}}}{\sqrt{N\left(1-P_{e_{B}}\right) V_{\mathcal{H}_{00_{B}}}+N P_{e_{B}} V_{\mathcal{H}_{01_{B}}}}}\right),
$$

where the index $B_{a p r}$ denotes approximation under the BPSK scenario. This equation simplifies (7), based on the fact that for a large number of samples, the expected number of correct and wrong decoded bits can be approximated with the help of the probability of correct $\left(1-P_{e_{B}}\right)$ and wrong bits $P_{e_{B}}$, respectively. Now, according to (27) the computation of $\varepsilon$ requires the inverse $\mathrm{Q}$ function which can be computed directly in most of the mathematical software packages.

\section{B. Probability of false alarm for QPSK signals}

In this subsection, we derive the $P_{F A}$ under the QPSK scenario. If we apply the algorithm proposed in Section II in under the QPSK scenario, the hypothesis test of (1) can be reformulated as in (28) at the top of the next page, where $n=0,1, \ldots, N-1$, the index $Q$ corresponds to the QPSK scenario, the hypothesis $\mathcal{H}_{00_{Q}}$ represents the case that the received signal is decoded correctly, while the hypotheses $\mathcal{H}_{01_{Q}}, \mathcal{H}_{02_{Q}}$ and $\mathcal{H}_{03_{Q}}$ correspond to the wrong decoding case and more specifically: i) $\mathcal{H}_{01_{Q}}$ : the real part is decoded wrongly and the imaginary part is correctly decoded, ii) $\mathcal{H}_{02_{Q}}$ : the real part is correctly decoded and the imaginary part is wrongly decoded and iii) $\mathcal{H}_{03_{Q}}$ : both the real and imaginary part are wrongly decoded.

Based on Theorem 1, for the derivation of the probability of false alarm under the BPSK scenario, the extension to QPSK case is straightforward and it is given as follows

$$
P_{F A_{Q}}=\sum_{k=0}^{2 N}\left(\begin{array}{c}
2 N \\
k
\end{array}\right) P_{k_{Q}} P_{e_{Q}}^{k}\left(1-P_{e_{Q}}\right)^{2 N-k},
$$

where $P_{k_{Q}}=P_{k_{B}}$ and $P_{e_{Q}}$ is the probability of bit error for QPSK, same as for BPSK, namely $P_{e_{Q}}=P_{e_{B}}$, while the factor 2 is due to the fact that a QPSK signal consists of two orthogonal BPSK ones. Furthermore, the corresponding approximated $P_{F A_{Q_{a p r}}}$ is given by (30) at the top of the next page, where $\mu_{\mathcal{H}_{00} 0_{Q}}=2 \mu_{\mathcal{H}_{00}{ }_{B}}$ and $V_{\mathcal{H}_{00} Q}=2 V_{\mathcal{H}_{00} 0_{B}}$ because both the real and imaginary part follow a truncated central chi-squared distribution, $\mu_{\mathcal{H}_{01} Q}=\mu_{\mathcal{H}_{00} B}+\mu_{\mathcal{H}_{01_{B}}}$ and $V_{\mathcal{H}_{01} Q}=V_{\mathcal{H}_{00_{B}}}+V_{\mathcal{H}_{01} B}$, because the real part follows a truncated non-central chi-squared distribution, while the imaginary part follows a truncated central chi-squared distribution, $\mu_{\mathcal{H}_{02} Q}=\mu_{\mathcal{H}_{00_{B}}}+\mu_{\mathcal{H}_{01_{B}}}$ and $V_{\mathcal{H}_{02} Q}=V_{\mathcal{H}_{00} B}+V_{\mathcal{H}_{01} B}$, because the real part follows a truncated central chi-squared distribution, while the imaginary part follows a truncated noncentral chi-squared distribution and finally, $\mu_{\mathcal{H}_{03} Q}=2 \mu_{\mathcal{H}_{01_{B}}}$ and $V_{\mathcal{H}_{03} Q}=2 V_{\mathcal{H}_{01_{B}}}$, because both the real and imaginary part follow a truncated non-central chi-squared distribution.

\section{Probability of detection}

In this subsection, we derive the probability of detection assuming that the primary signal follows a Gaussian distribution with zero mean and variance $\sigma_{P U}^{2}$. This is a valid assumption, e.g. with an OFDM signal, where independent data streams are used for the modulation of each carrier [34]. Furthermore, it is customary to assume that $x_{p}$ is Gaussian-distributed, because the modulation and generally the symbols of the primary user are unknown [13]. Similar signal modeling is frequently used in cognitive radio literature, e.g. [35]-[37]. Therefore, under the scenario that the SU signal is BPSK modulated, the theoretical expression for the $P_{D}$ is also given by (27) by substituting $\sigma_{w}^{2}$ with $\sigma_{w}^{2}+\sigma_{P U}^{2}$ in the related parts. Specifically, the $P_{D}$ is given by

$$
P_{D_{B_{a p}}}=Q\left(\frac{\varepsilon-N\left(1-P_{e_{B}}^{\prime}\right) \mu_{\mathcal{H}_{10_{B}}}-N P_{e_{B}}^{\prime} \mu_{\mathcal{H}_{11_{B}}}}{\sqrt{N\left(1-P_{e_{B}}^{\prime}\right) V_{\mathcal{H}_{10_{B}}}+N P_{e_{B}}^{\prime} V_{\mathcal{H}_{11_{B}}}}}\right),
$$




$$
\begin{aligned}
& \mathcal{H}_{0_{Q}}:\left\{\begin{array}{l}
\mathcal{H}_{00_{Q}}: y(n)=\mathcal{R}\{w(n)\}+\mathcal{I}\{w(n)\}, \\
\mathcal{H}_{01_{Q}}: y(n)=2 h \mathcal{R}\{s(n)\}+\mathcal{R}\{w(n)\}+\mathcal{I}\{w(n)\}, \\
\mathcal{H}_{02_{Q}}: y(n)=2 h \mathcal{I}\{s(n)\}+\mathcal{R}\{w(n)\}+\mathcal{I}\{w(n)\}, \\
\mathcal{H}_{03_{Q}}: y(n)=2 h \mathcal{R}\{s(n)\}+2 h \mathcal{I}\{s(n)\}+\mathcal{R}\{w(n)\}+\mathcal{I}\{w(n)\},
\end{array}\right. \\
& P_{F A_{Q_{a p r}}}=Q\left(\frac{\varepsilon-N\left(1-P_{e_{Q}}\right)^{2} \mu_{\mathcal{H}_{00} Q}-N\left(1-P_{e_{Q}}\right) P_{e_{Q}}\left(\mu_{\mathcal{H}_{01}{ }_{Q}}+\mu_{\mathcal{H}_{02_{Q}}}\right)-N P_{e_{Q}}^{2} \mu_{\mathcal{H}_{03} Q}}{\sqrt{N\left(1-P_{e_{Q}}\right)^{2} V_{\mathcal{H}_{00} Q}+N\left(1-P_{e_{Q}}\right) P_{e_{Q}}\left(V_{\mathcal{H}_{01} Q}+V_{\mathcal{H}_{02} Q}\right)+N P_{e_{Q}}^{2} V_{\mathcal{H}_{03}}}}\right) .
\end{aligned}
$$

where the mean $\mu_{\mathcal{H}_{10_{B}}}, \mu_{\mathcal{H}_{11_{B}}}$ and variance $V_{\mathcal{H}_{10_{B}}}, V_{\mathcal{H}_{11_{B}}}$ are defined by (23), (24), (25) and (26), respectively, with the difference being that now, the probability of bit error and the interval of interest are not anymore related to $P_{e_{B}}=$ $Q\left(\sqrt{\frac{\gamma P_{s}}{\sigma_{w}^{2}}}\right)$ and $P_{t}=\frac{\gamma P_{s}}{\sigma_{w}^{2}+\sigma_{P U}^{2}}$, but they are based on the following parameters: $P_{e_{B}}^{\prime}=Q\left(\sqrt{\frac{\gamma P_{s}}{\sigma_{w}^{2}+\sigma_{P U}^{2}}}\right), P_{d}=\frac{\gamma P_{s}}{\sigma_{w}^{2}+\sigma_{P U}^{2}}$. Similarly, the $P_{D}$ under the scenario that the SU signal is QPSK modulated is given by (30) by substituting again $\sigma_{w}^{2}$ with $\sigma_{w}^{2}+\sigma_{P U}^{2}$ in the related parts.

As mentioned, in this paper, we derive the $P_{D}$ under the assumption that the primary signal is Gaussian-distributed. Deriving the $P_{D}$ for the case that the primary signal has different distribution is a valuable idea for future studies.

\section{PRobability of FAlSE ALARM FOR M-QAM}

In the previous section, we discussed the derivation of the $P_{F A}$ and $P_{D}$ under the BPSK and QPSK scenarios. However, in this section, we generalize our work to higher modulation schemes, and particularly, we examine the $P_{F A}$ for M-QAM modulated signals. The derivation of the $P_{D}$ is straightforward as in Section III. Furthermore, we should note that here we focus on the derivation of the approximated $P_{F A}$, because as mentioned earlier, the calculation of the detection threshold $\varepsilon$ through the accurate $P_{F A}$ is complicated, especially as $N$ increases and the situation becomes more complex under the M-QAM scenario due to the fact that under the wrong decoding case the estimated symbol can be anyone of $M-1$ possible symbols.

The approximated probability of false alarm for the M-QAM case $\left(P_{F A_{M-Q a p r}}\right)$ is defined by

$$
\begin{aligned}
& P_{F A_{M-Q_{a p r}}}= \\
& =Q\left(\frac{\varepsilon-N \sum_{k=1}^{M} \sum_{t=1}^{M} P\left(s=s_{k}\right) P\left(\hat{s}=s_{t} \mid s=s_{k}\right) \mu_{\mathcal{H}_{0, k, t}}}{\sqrt{N \sum_{k=1}^{M} \sum_{t=1}^{M} P\left(s=s_{k}\right) P\left(\hat{s}=s_{t} \mid s=s_{k}\right) V_{\mathcal{H}_{0, k, t}}}}\right)
\end{aligned}
$$

where $s_{k}$ denotes the transmitted symbol, $s_{t}$ denotes the estimated symbol, while $\mu_{\mathcal{H}_{0, k, t}}$ and $V_{\mathcal{H}_{0, k, t}}$ represent, respectively, the mean and variance of the test statistic of (6) under each case of correct or wrong decoding under $\mathcal{H}_{0}$ hypothesis, expressed as follows:

$$
\begin{aligned}
& \mu_{\mathcal{H}_{0, k, t}}=\mu_{\frac{\mathcal{R}\left\{s_{k}\right\}-\mathcal{R}\left\{s_{t}\right\}}{\sqrt{P_{s}}}}+\mu_{\mathcal{I}\left\{s_{k}\right\}-\mathcal{I}\left\{s_{t}\right\}}, \\
& V_{\mathcal{H}_{0, k, t}}=\frac{V_{\mathcal{R}\left\{s_{k}\right\}-\mathcal{R}\left\{s_{t}\right\}}}{\sqrt{P_{s}}}+V_{\mathcal{I}\left\{s_{k}\right\}-\mathcal{I}\left\{s_{t}\right\}},
\end{aligned}
$$

where $\quad \mu_{\frac{\mathcal{R}\left\{s_{k}\right\}-\mathcal{R}\left\{s_{t}\right\}}{\sqrt{P_{s}}}}, \quad \mu_{\frac{\mathcal{I}\left\{s_{k}\right\}-\mathcal{I}\left\{s_{t}\right\}}{\sqrt{P_{s}}}}, \quad \frac{V_{\mathcal{R}\left\{s_{k}\right\}-\mathcal{R}\left\{s_{t}\right\}}}{\sqrt{P_{s}}}$, $V_{\frac{\mathcal{I}\left\{s_{k}\right\}-\mathcal{I}\left\{s_{t}\right\}}{\sqrt{P_{s}}}}$, are derived by Theorems 4 and 5 .

For the case of correct decoding, namely when $k=t$, it is obvious that (33) and (34) are expressed as follows: $\mu_{\mathcal{H}_{0, k, t}}=$ $\mu_{0}+\mu_{0}$ and $V_{\mathcal{H}_{0, k, t}}=V_{0}+V_{0}$. Therefore, the derivation of the mean and variance under the scenario of correct decoding for M-QAM signals is required and it is given by Theorem 4 .

Theorem 4: Consider a secondary user with one receive antenna, which collects a number of samples $N$. The SU-Rx decodes the samples, removes them from the total received signal and applies the Energy Detector in the remaining signal. Then, the mean and variance of $T\left(y^{\prime} \mid \mathcal{H}_{0, k=t, t=k}\right)$ can be respectively defined as follows:

$$
\begin{aligned}
& \mu_{\mathcal{H}_{0, k=t, t=k}}=2 \mu_{0}=E\left\{\left|y^{\prime}\right|^{2} \mid \mathcal{H}_{0, k=t, t=k}\right\} \\
& =2 E\left\{\mathcal{R}^{2}\left\{y^{\prime}\right\} \mid 0 \leq \mathcal{R}^{2}\{w\} \leq P_{t}\right\} P_{1} \\
& +2 \frac{2}{\sqrt{M}} E\left\{\mathcal{R}^{2}\left\{y^{\prime}\right\} \mid \mathcal{R}^{2}\{w\} \geq P_{t}\right\} P_{2}, \\
& V_{\mathcal{H}_{0, k=t, t=k}}=2 V_{0}=V\left\{\left|y^{\prime}\right|^{2} \mid \mathcal{H}_{0, k=t, t=k}\right\} \\
& =2 V\left\{\mathcal{R}^{2}\left\{y^{\prime}\right\} \mid 0 \leq \mathcal{R}^{2}\{w\} \leq P_{t}\right\} P_{1} \\
& +2 \frac{2}{\sqrt{M}} V\left\{\mathcal{R}^{2}\left\{y^{\prime}\right\} \mid \mathcal{R}^{2}\{w\} \geq P_{t}\right\} P_{2} \\
& +2\left(E\left\{\mathcal{R}^{2}\left\{y^{\prime}\right\} \mid 0 \leq \mathcal{R}^{2}\{w\} \leq P_{t}\right\}\right)^{2} P_{1} P_{2} \\
& +2 \frac{2}{\sqrt{M}}\left(E\left\{\mathcal{R}^{2}\left\{y^{\prime}\right\} \mid \mathcal{R}^{2}\{w\} \geq P_{t}\right\}\right)^{2} P_{1} P_{2} \\
& -2 \frac{4}{\sqrt{M}} E\left\{\mathcal{R}^{2}\left\{y^{\prime}\right\} \mid 0 \leq \mathcal{R}^{2}\{w\} \leq P_{t}\right\} \\
& \times E\left\{\mathcal{R}^{2}\left\{y^{\prime}\right\} \mid \mathcal{R}^{2}\{w\} \geq P_{t}\right\} P_{1} P_{2},
\end{aligned}
$$

where $\mathcal{H}_{0, k=t, t=k}$ represents the case of correct decoding for M-QAM signals under $\mathcal{H}_{0}$ hypothesis, $M$ denotes the size of the constellation, $P_{\mathrm{C}_{\mathrm{M}-\mathrm{Q}}}$ corresponds to the probability of correct symbol for M-QAM modulation [24] and $P_{1}, P_{2}$ are respectively obtained by (37) and (38) at the top of the next page.

Proof: The proof of (35) is presented in Appendix C, while the proof of (36) is similar to that of (35). 


$$
\begin{gathered}
P_{1}=P\left(-\sqrt{P_{t}} \leq \mathcal{R}\{w\} \leq \sqrt{P_{t}}\right)\left[\begin{array}{c}
P\left(-\sqrt{P_{t}} \leq \mathcal{I}\{w\} \leq \sqrt{P_{t}}\right)+ \\
+2 P\left(\mathcal{I}\{w\} \geq \sqrt{P_{t}}\right) / \sqrt{M}
\end{array}\right] / P_{\mathrm{c}_{-} \mathrm{M}-\mathrm{Q}}, \\
P_{2}=P\left(\mathcal{R}\{w\} \geq \sqrt{P_{t}}\right)\left[\begin{array}{c}
P\left(-\sqrt{P_{t}} \leq \mathcal{I}\{w\} \leq \sqrt{P_{t}}\right)+ \\
+2 P\left(\mathcal{I}\{w\} \geq \sqrt{P_{t}}\right) / \sqrt{M}
\end{array}\right] / P_{\mathrm{c}_{\mathrm{M}-\mathrm{Q}}}
\end{gathered}
$$

Furthermore, the derivation of the mean and variance under the scenario of wrong decoding for M-QAM signals is necessary and it is given by Theorem 5 .

Theorem 5: Consider a secondary user with one receive antenna, which collects a number of samples $N$. The SU-Rx decodes the samples, removes them from the total received signal and applies the Energy Detector in the remaining signal. Then, the mean and variance of $T\left(y^{\prime} \mid \mathcal{H}_{0, \mathrm{k} \neq \mathrm{t}, \mathrm{t} \neq \mathrm{k}}\right)$ are defined by (33) and (34), respectively, where $\frac{\mu_{\frac{\mathcal{R}\left\{s_{k}\right\}-\mathcal{R}\left\{s_{t}\right\}}{\sqrt{P_{s}}}}}{{ }^{2}} \mu_{2 l}$ and

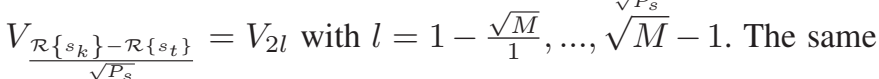
range is also valid for $\mu_{\frac{\mathcal{I}\left\{s_{k}\right\}-\mathcal{I}\left\{s_{t}\right\}}{\sqrt{P_{s}}}}$ and $V_{\frac{\mathcal{I}\left\{s_{k}\right\}-\mathcal{I}\left\{s_{t}\right\}}{\sqrt{P_{s}}}}$. More specifically the mean $\mu_{2 l}$, for the whole range, is derived as follows:

$$
\begin{aligned}
& \mu_{2 r}=\left(1+\frac{2 r}{\sqrt{M}}\right) \\
& \times E\left\{\left|\mathcal{R}\left\{y^{\prime}\right\}\right|^{2}|0 \leq| 2 r \sqrt{P_{t}}+\left.\mathcal{R}\{w\}\right|^{2} \leq P_{t}\right\} P_{3} \\
& +\frac{2}{\sqrt{M}} E\left\{\left|\mathcal{R}\left\{y^{\prime}\right\}\right|^{2}|| 2 r \sqrt{P_{t}}+\left.\mathcal{R}\{w\}\right|^{2} \geq P_{t}\right\} P_{4},
\end{aligned}
$$

$$
\begin{aligned}
& \mu_{2 d}= \\
& =E\left\{\left|2 d \sqrt{P_{t}}+\mathcal{R}\{w\}\right|^{2}|0 \leq| 2 d \sqrt{P_{t}}+\left.\mathcal{R}\{w\}\right|^{2} \leq P_{t}\right\} \\
& \times P_{5} . \\
& \mu_{2 f}=E\left\{\left|\mathcal{R}\left\{y^{\prime}\right\}\right|^{2}|0 \leq| 2 f \sqrt{P_{t}}+\left.\mathcal{R}\{w\}\right|^{2} \leq P_{t}\right\} P_{6} \\
& \quad+\frac{2}{\sqrt{M}} E\left\{\left|\mathcal{R}\left\{y^{\prime}\right\}\right|^{2}|| 2 f \sqrt{P_{t}}+\left.\mathcal{R}\{w\}\right|^{2} \geq P_{t}\right\} P_{7},
\end{aligned}
$$$$
\mu_{2 q}=\left(2-\frac{2 q}{\sqrt{M}}\right)
$$$$
\times E\left\{\left|\mathcal{R}\left\{y^{\prime}\right\}\right|^{2}|0 \leq| 2 q \sqrt{P_{t}}+\left.\mathcal{R}\{w\}\right|^{2} \leq P_{t}\right\} P_{8}
$$$$
+\frac{2}{\sqrt{M}} E\left\{\left|\mathcal{R}\left\{y^{\prime}\right\}\right|^{2}|| 2 q \sqrt{P_{t}}+\left.\mathcal{R}\{w\}\right|^{2} \geq P_{t}\right\} P_{9},
$$

where $r=1-\frac{\sqrt{M}}{2}, \ldots,-1, d=1, \ldots, \frac{\sqrt{M}}{2}-1, f=\frac{\sqrt{M}}{2}$ and $q=\frac{\sqrt{M}}{2}+1, \ldots, \sqrt{M}-1$, while the variance $V_{2 r}$ is obtained as follows:

$V_{2 r}=\left(1+\frac{2 r}{\sqrt{M}}\right)$

$$
\begin{aligned}
& \times V\left\{\left|\mathcal{R}\left\{y^{\prime}\right\}\right|^{2}|0 \leq| 2 r \sqrt{P_{t}}+\left.\mathcal{R}\{w\}\right|^{2} \leq P_{t}\right\} P_{3} \\
& +\frac{2}{\sqrt{M}} V\left\{\left|\mathcal{R}\left\{y^{\prime}\right\}\right|^{2}|| 2 r \sqrt{P_{t}}+\left.\mathcal{R}\{w\}\right|^{2} \geq P_{t}\right\} P_{4} \\
& +\left(1+\frac{2 r}{\sqrt{M}}\right) \\
& \times\left(E\left\{\left|\mathcal{R}\left\{y^{\prime}\right\}\right|^{2}|0 \leq| 2 r \sqrt{P_{t}}+\left.\mathcal{R}\{w\}\right|^{2} \leq P_{t}\right\}\right)^{2} P_{3} P_{4} \\
& +\frac{2}{\sqrt{M}}\left(E\left\{\left|\mathcal{R}\left\{y^{\prime}\right\}\right|^{2}|| 2 r \sqrt{P_{t}}+\left.\mathcal{R}\{w\}\right|^{2} \geq P_{t}\right\}\right)^{2} P_{3} P_{4} \\
& -2\left(1+\frac{2 r}{\sqrt{M}}\right) \frac{2}{\sqrt{M}} \\
& \times E\left\{\left|\mathcal{R}\left\{y^{\prime}\right\}\right|^{2}|0 \leq| 2 r \sqrt{P_{t}}+\left.\mathcal{R}\{w\}\right|^{2} \leq P_{t}\right\} \\
& \times E\left\{\left|\mathcal{R}\left\{y^{\prime}\right\}\right|^{2}|| 2 r \sqrt{P_{t}}+\left.\mathcal{R}\{w\}\right|^{2} \geq P_{t}\right\} P_{3} P_{4},
\end{aligned}
$$

where $r=1-\frac{\sqrt{M}}{2}, \ldots,-1$, while the probabilities $P_{3}, P_{4}, P_{5}, P_{6}, P_{7}, P_{8}$ and $P_{9}$ are given by

$$
\begin{aligned}
& P_{3}=\frac{P\left(-\sqrt{P_{t}}-2 r \sqrt{P_{t}} \leq \mathcal{R}\{w\} \leq \sqrt{P_{t}}-2 r \sqrt{P_{t}}\right)}{P_{w_{M-Q}}} \\
& P_{4}=\frac{P\left(\mathcal{R}\{w\} \geq \sqrt{P_{t}}-2 r \sqrt{P_{t}}\right)}{P_{w_{M-Q}}} \\
& P_{5}=\frac{P\left(-\sqrt{P_{t}}-2 d \sqrt{P_{t}} \leq \mathcal{R}\{w\} \leq \sqrt{P_{t}}-2 d \sqrt{P_{t}}\right)}{P_{w_{M-Q}}} \\
& P_{6}=\frac{P\left(-\sqrt{P_{t}}-2 f \sqrt{P_{t}} \leq \mathcal{R}\{w\} \leq \sqrt{P_{t}}-2 f \sqrt{P_{t}}\right)}{P_{w_{M-Q}}} \\
& P_{7}=\frac{P\left(\mathcal{R}\{w\} \geq \sqrt{P_{t}}-2 f \sqrt{P_{t}}\right)}{P_{w_{M-Q}}} \\
& P_{8}=\frac{P\left(-\sqrt{P_{t}}-2 q \sqrt{P_{t}} \leq \mathcal{R}\{w\} \leq \sqrt{P_{t}}-2 q \sqrt{P_{t}}\right)}{P_{w_{M-Q}}} \\
& P_{9}=\frac{P\left(\mathcal{R}\{w\} \geq \sqrt{P_{t}}-2 q \sqrt{P_{t}}\right)}{P_{w_{M-Q}}},
\end{aligned}
$$

while $\mathcal{H}_{0, k \neq t, t \neq k}$ represents the case of wrong decoding for M-QAM signals under $\mathcal{H}_{0}$ hypothesis and $P_{w_{M-Q}}$ denotes the probability of wrong symbol detection for M-QAM modulation [24]. Finally, we should mention that the derivation of the variances $V_{2 d}, V_{2 f}$ and $V_{2 q}$ is not provided in this paper due to lack of space, but they can be defined following the same methodology as for (43).

Proof: The proof of Theorem 5 is presented in Appendix D. 


$$
T\left(\mathbf{y} \mid \mathcal{H}_{0_{B}}\right) \sim \mathcal{N}\left(N\left(1-P_{e_{B}}\right) \mu_{\mathcal{H}_{00_{B}}}+N P_{e_{B}} \mu_{\mathcal{H}_{01_{B}}}, N\left(1-P_{e_{B}}\right) V_{\mathcal{H}_{00_{B}}}+N P_{e_{B}} V_{\mathcal{H}_{01_{B}}}\right)
$$

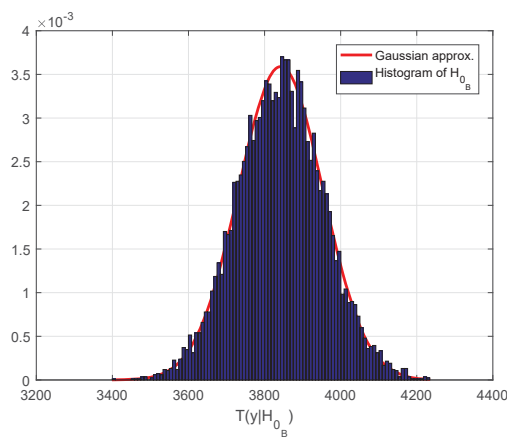

(a)

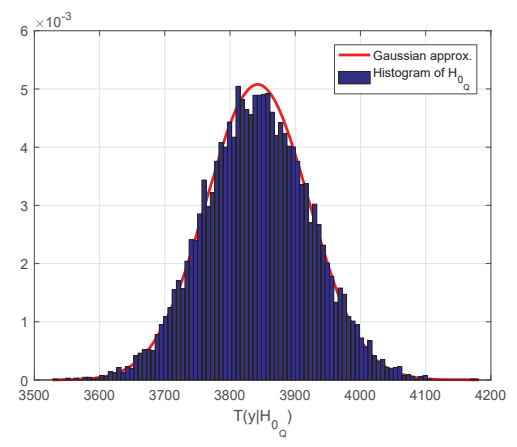

(b)

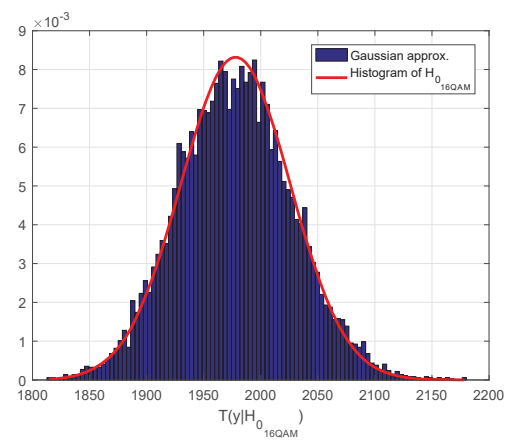

(c)

Fig. 6: Theoretical and empirical distributions of (a) $T\left(\mathbf{y} \mid \mathcal{H}_{0_{B}}\right)$, (b) $T\left(\mathbf{y} \mid \mathcal{H}_{0_{Q}}\right)$ and (c) $T\left(\mathbf{y} \mid \mathcal{H}_{0_{16-Q A M}}\right)$ with $N=2000$, $E_{s}=10 \mathrm{~dB}$ and $\sigma_{w}^{2}=2 \mathrm{~dB}$.

\section{NUMERICAL RESULTS}

In this section, we present simulation results in order to i) verify the derived expressions of (27), (30) and (32) for the $P_{F A}$, ii) compare our proposed system with the conventional one in terms of the throughput, iii) evaluate the detection reliability of the "ED with imperfect signal cancellation (EDISC)" for the case of perfect channel estimation and iv) examine the $P_{D}$ of our proposed method taking into account the channel estimation error.

\section{A. Evaluation of the probability of false alarm}

We should mention that under the assumption of a large number of samples $N$ and the use of the CLT, the relationship between the $P_{F A}$ and the corresponding distribution of the test statistic is the following: $T(\mathbf{y}) \sim \mathcal{N}(\mu, V)=>P_{F A}=$ $Q\left(\frac{\varepsilon-\mu}{\sqrt{V}}\right)$, where $\mathcal{N}(\mu, V)$ denotes a Gaussian distribution with mean $\mu$ and variance $V$. Therefore, the verification of the $P_{F A}$ under the BPSK (27), QPSK (30) and M-QAM (32) scenarios can be obtained through the verification of the distribution of the test statistic under the BPSK $\left(T\left(\mathbf{y} \mid \mathcal{H}_{0_{B}}\right)\right)$, QPSK $\left(T\left(\mathbf{y} \mid \mathcal{H}_{0_{Q}}\right)\right)$ and 16-QAM $\left(T\left(\mathbf{y} \mid \mathcal{H}_{0_{16-Q A M}}\right)\right)$ scenarios. The distribution of $T\left(\mathbf{y} \mid \mathcal{H}_{0_{B}}\right)$ is given by (45) at the top of the page. With similar manner, we can derive the distribution of $T\left(\mathbf{y} \mid \mathcal{H}_{0_{Q}}\right)$ and $T\left(\mathbf{y} \mid \mathcal{H}_{0_{16-Q A M}}\right)$, which we will not show here due to lack of space.

Figures $6 \mathrm{a}, 6 \mathrm{~b}$ and $6 \mathrm{c}$ depict the histogram obtained from 10000 Monte-Carlo realizations of $T\left(\mathbf{y} \mid \mathcal{H}_{0_{B}}\right), T\left(\mathbf{y} \mid \mathcal{H}_{0_{Q}}\right)$ and $T\left(\mathbf{y} \mid \mathcal{H}_{0_{16-Q A M}}\right)$, respectively, where the number of measurement samples is set to $N=2000$. In this figure, we show that the CLT provides good approximation for the distribution of $T\left(\mathbf{y} \mid \mathcal{H}_{0_{B}}\right), T\left(\mathbf{y} \mid \mathcal{H}_{0_{Q}}\right)$ and $T\left(\mathbf{y} \mid \mathcal{H}_{0_{16-Q A M}}\right)$, verifying with this way the Gaussian approximations of (27), (30) and (32) for the $P_{F A}$.

\section{B. Performance analysis with respect to throughput}

In this subsection, we compare our proposed scheme with te conventional one in terms of the average achievable throughput.
As we follow the same model as in [23], we can use the same approach for the evaluation of the throughput, replacing the $P_{F A}$ and $P_{D}$ under the case of perfect signal cancellation with our deriving expressions for the $P_{F A}$ and $P_{D}$ under the case of imperfect signal cancellation. For conciseness, we refer the readers to [23] for the details of the throughput derivation. The frame duration is $T=100 \mathrm{~ms}$, the sampling frequency $f=6 \mathrm{MHz}$ and for both cases, we assume a target probability of detection $P_{D}=0.9$, while the secondary transmit and primary received SNR are considered as $S N R_{S U}=7 \mathrm{~dB}$ and $S N R_{P U}=-20 \mathrm{~dB}$.

Figure 7 depicts the average achievable throughput versus the sensing time $\tau$, where it is obvious that the proposed cognitive radio systems exhibits higher average achievable throughput than the conventional one despite the fact of considering imperfect signal cancellation.

\section{Performance analysis with respect to $P_{D}$}

In this paper, we assume that the PU is either absent or present for a long period as in fixed networks, e.g. TV channels and backhaul networks. Therefore, in this section, we present simulation results to analyze the detection reliability of the "ED with imperfect signal cancellation (EDISC)" for the frame that the simultaneous spectrum sensing and data transmission takes place. We compare this scheme with the "ED with perfect signal cancellation (EDPSC)" as proposed in [23] and with the case that the SU-Rx informs the SU-Tx about the status of the PU by using a "Conventional ED (CED)" as given by (5) in [38], where there is no need for decoding, and hence signal cancellation. For simplicity, we assume that the PU follows a Gaussian distribution with zero mean and variance $\sigma_{P U}^{2}$, while the transmitted signal from the SU-TX is BPSK, QPSK, 16-QAM or 64-QAM modulated. Furthermore, the channel is assumed to be of unit power, the probability of false alarm is set to $P_{F A}=0.1$, while the received PU Signal to Interference plus Noise Ratio (SINR) at the SU-Rx $\left(S I N R_{P U}=\frac{\sigma_{P U}^{2}}{P_{s}+\sigma_{w}^{2}}\right)$ 


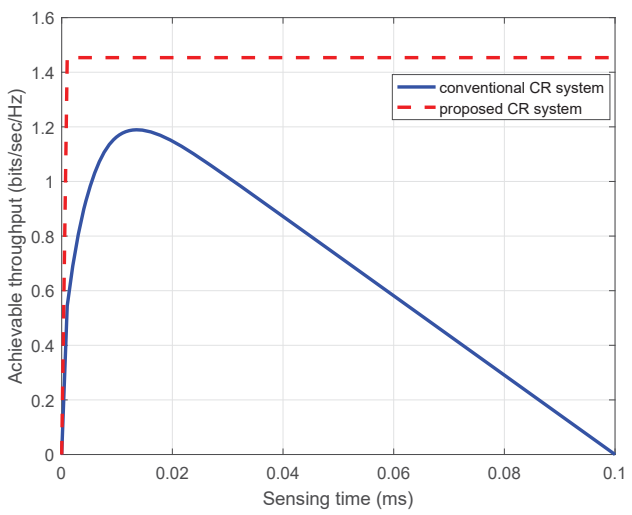

(a) Probability of PU being active $P\left(\mathcal{H}_{0}\right)=0.5$.

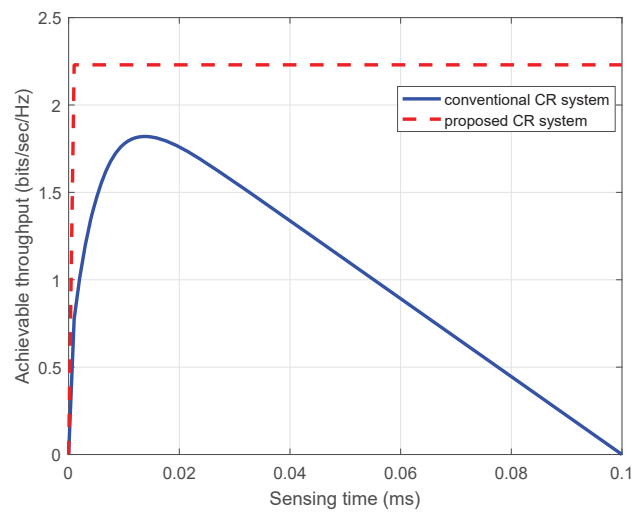

(b) Probability of PU being active $P\left(\mathcal{H}_{0}\right)=0.8$.

Fig. 7: Average achievable throughput of the proposed and conventional cognitive radio system versus the sensing time with secondary transmit SNR, $S N R_{S U}=7 d B$, received primary SNR, $S N R_{P U}=-20 \mathrm{~dB}$ and target detection probability $P_{D}=0.9$.

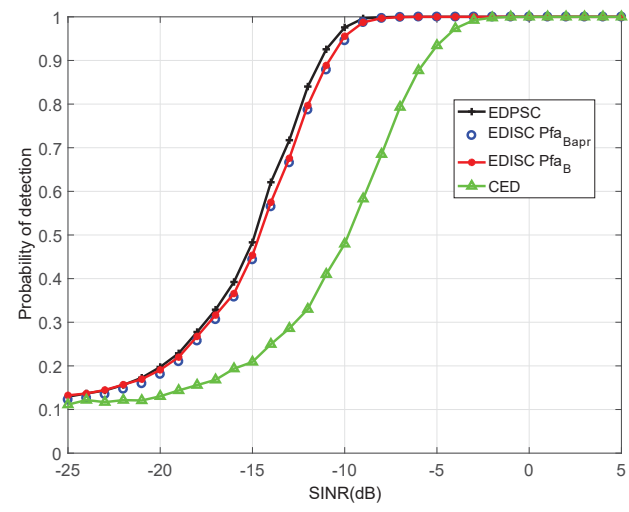

Fig. 8: $P_{D}$ versus the SINR of the PU, under the BPSK scenario, for $N=100, E_{s}=10 \mathrm{~dB}$ and $\sigma_{w}^{2}=2 \mathrm{~dB}$.

varies from -25 to $5 \mathrm{~dB}$. Here, we should mention that by saying interference we mean the presence of the SU-Tx.

Figures $8,9,10$ and 11 depict the $P_{D}$ as a function of the SINR of the PU comparing the aforementioned three techniques: i) CED, ii) EDPSC and iii) EDISC. The number of samples is set $N=100$ and it is observed that our proposed detection scheme provides significantly better detection performance than the CED for all the cases. Furthermore, it is observed that the EDISC approaches the detection performance of the EDPSC performing slightly worse, under the BPSK and QPSK scenario, while it presents inferior performance for 16-QAM and 64QAM compared to ones for BPSK/QPSK, but still better. This can be explained by the fact that our technique considers a more realistic scenario taking into account the decoding errors and the probability of correct decision deteriorates as we employ higher order constellations (especially for low SINR values). Moreover, Figures 8 and 9 show that the performance of the $P_{D}$ based on the calculated decision threshold related to the approximated $P_{F A}$ (i.e. (27) and (30) for Figure 8 and Figure 9 , respectively) is very close to the $P_{D}$ based on the calculated decision threshold related to the more accurate $P_{F A}$ (i.e. (7) and (29) for Figure 8 and Figure 9, respectively), validating

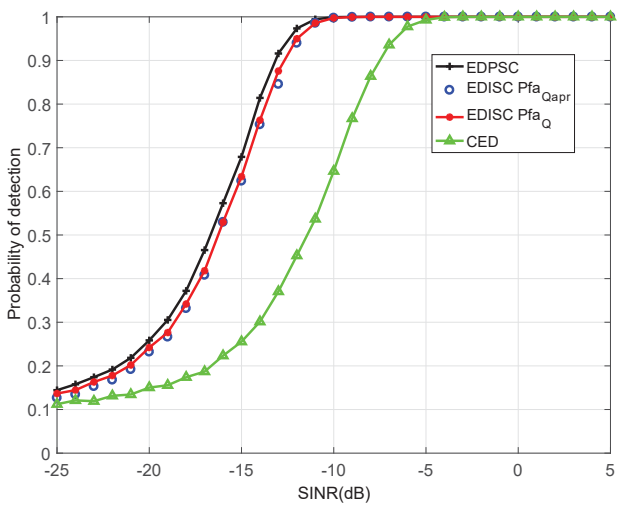

Fig. 9: $P_{D}$ versus the SINR of the PU, under the QPSK scenario, for $N=100, E_{s}=10 \mathrm{~dB}$ and $\sigma_{w}^{2}=2 \mathrm{~dB}$.

the reliability of (27) and (30), respectively.

Finally, from Figure 12 is observed that the difference in the detection performance between the simulation and approximated results decreases as the number of samples increases. The reason is that our theoretical approximated expression is based on the CLT which requires a large number of samples for better reliability.

\section{Performance analysis with respect to channel estimation error}

In the following experiment, we evaluate the performance of the EDISC with respect to the channel uncertainty under the BPSK case. The estimated channel $\hat{h}$ can be modeled as $\hat{h}=h+\epsilon$, where the channel estimation error $\epsilon$ follows a Gaussian distribution with zero mean and variance $\sigma_{\epsilon}^{2}$ [38][39]. Therefore, in the simulations, the channel estimation error varies in each realization.

Figure 13 depicts the $P_{D}$ as a function of the SINR of the PU for the BPSK case considering imperfect channel estimation. This figure shows that the channel uncertainty degrades the detection performance of the EDISC. Furthermore, we see that 


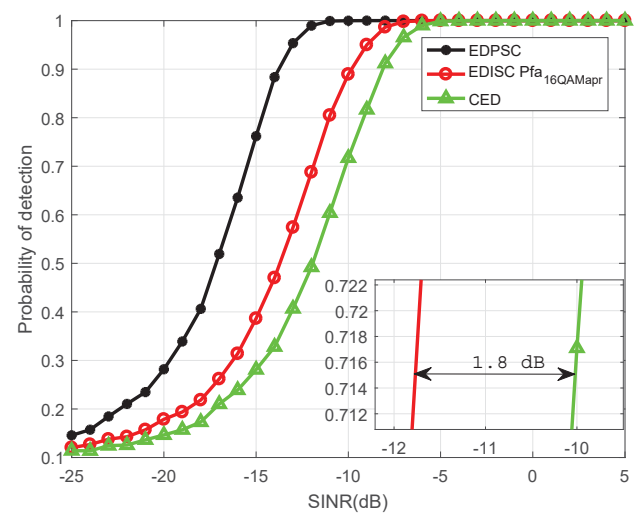

Fig. 10: $P_{D}$ versus the SINR of the PU, under the 16-QAM scenario, for $N=100, E_{s}=10 \mathrm{~dB}$ and $\sigma_{w}^{2}=2 \mathrm{~dB}$.

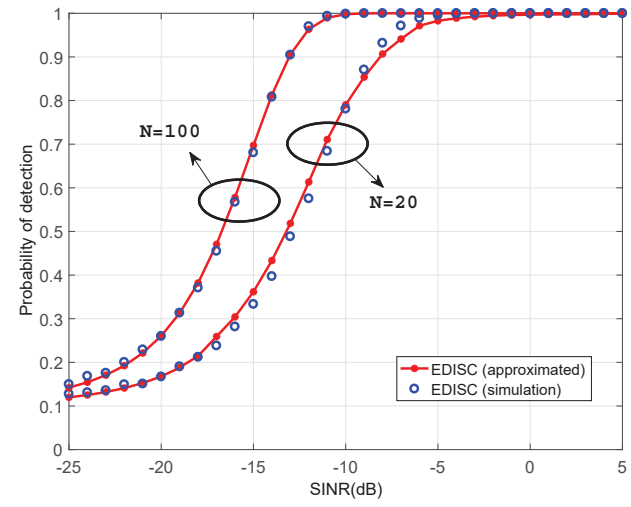

Fig. 12: Comparison between the approximated and simulated performance for the proposed ED, under the QPSK scenario for $E_{s}=7 \mathrm{~dB}$ and $\sigma_{w}^{2}=0 \mathrm{~dB}$.

when the channel estimation is more accurate $\sigma_{\epsilon}^{2}=0.002$, the effect of the channel estimation error reduces, but our detector, still, performs well for moderate channel uncertainty $\sigma_{\epsilon}^{2}=0.02$

\section{CONCLUSIONS}

In this paper, we investigated the idea of simultaneous spectrum sensing and data transmission considering imperfect signal cancellation in the data domain. We analyzed how the decoding errors affect the detection reliability of the system and derived the analytical expressions for the probability of false alarm assuming digitally modulated signals (i.e. BPSK, QPSK, M-QAM). Furthermore, we presented a detailed analysis around the distribution of the sum of $N$ truncated central or non-central chi-squared random variables. Finally, the numerical results showed that the detection performance of our proposed scheme is considerably better than the conventional ED, verifying in this way the accuracy of the proposed study. Future works include i) the derivation of the analytical expressions for the $P_{F A}$ under the channel estimation errors, ii) the derivation of the $P_{D}$ for the case that the primary signal has different distribution than Gaussian and iii) the adaptation of our scheme in a more dynamic traffic pattern.

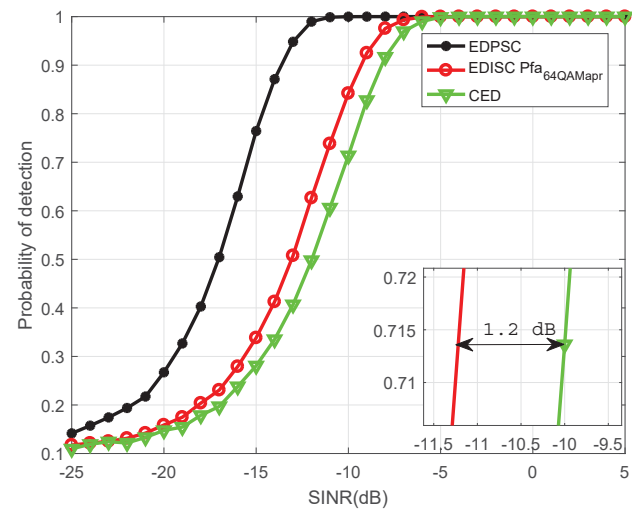

Fig. 11: $P_{D}$ versus the SINR of the PU, under the 64-QAM scenario, for $N=100, E_{s}=10 \mathrm{~dB}$ and $\sigma_{w}^{2}=2 \mathrm{~dB}$.

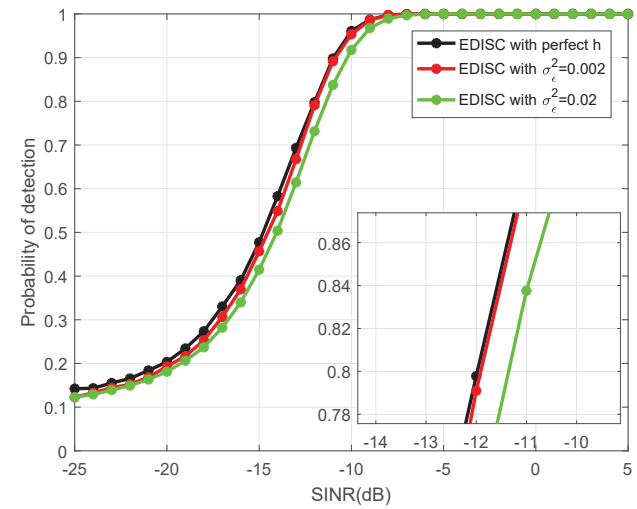

Fig. 13: $P_{D}$ versus the SINR of the PU, for the BPSK case considering channel uncertainty, for $N=100, E_{s}=10 \mathrm{~dB}$ and $\sigma_{w}^{2}=2 \mathrm{~dB}$.

\section{APPENDIX A}

DERIVATION OF THE MEAN $\mu_{\mathcal{H}_{00_{B}}}$

For the derivation of the mean $\mu_{\mathcal{H}_{00_{B}}}$, we use the law of total expectation [40] as follows:

$$
\begin{aligned}
& E\left\{\left.\left|y^{\prime}\right|\right|^{2} \mid \mathcal{H}_{00_{B}}\right\}= \\
& =E\left\{\left|y^{\prime}\right|^{2} \mid s=+\sqrt{P_{s}}, \hat{s}=+\sqrt{P_{s}}\right\} \\
& \times P\left(s=+\sqrt{P_{s}}\right) P\left(\hat{s}=+\sqrt{P_{s}} \mid c_{B}\right) \\
& +E\left\{\left|y^{\prime}\right|^{2} \mid s=-\sqrt{P_{s}}, \hat{s}=-\sqrt{P_{s}}\right\} \\
& \times P\left(s=-\sqrt{P_{s}}\right) P\left(\hat{s}=-\sqrt{P_{s}} \mid c_{B}\right),
\end{aligned}
$$

where the expression of (46) can be extended as follows:

$$
\begin{aligned}
& E\left\{\left|y^{\prime}\right|^{2} \mid \mathcal{H}_{00_{B}}\right\}= \\
& =\frac{1}{2} E\left\{\left|y^{\prime}\right|^{2} \mid w \geq-\sqrt{P_{t}}\right\} P\left(w \geq-\sqrt{P_{t}} \mid c_{B}\right) \\
& +\frac{1}{2} E\left\{\left|y^{\prime}\right|^{2} \mid w \leq+\sqrt{P_{t}}\right\} P\left(w \leq+\sqrt{P_{t}} \mid c_{B}\right) .
\end{aligned}
$$


However, the remaining noise under $\mathcal{H}_{00_{B}}$ follows a truncated normal distribution in the following intervals: i) $w \leq-\sqrt{P_{t}}$, ii) $-\sqrt{P_{t}} \leq w \leq \sqrt{P_{t}}$ and iii) $w \geq \sqrt{P_{t}}$. Thus, exploiting the symmetry of the BPSK constellation, (47) can be further expressed by

$$
\begin{aligned}
& E\left\{\left|y^{\prime}\right|^{2} \mid \mathcal{H}_{00_{B}}\right\}= \\
& =E\left\{\left|y^{\prime}\right|^{2} \mid-\sqrt{P_{t}} \leq w \leq \sqrt{P_{t}}\right\} \\
& \times P\left(-\sqrt{P_{t}} \leq w \leq \sqrt{P_{t}} \mid c_{B}\right) \\
& +E\left\{\left|y^{\prime}\right|^{2} \mid w \geq \sqrt{P_{t}}\right\} \\
& \times P\left(w \geq+\sqrt{P_{t}} \mid c_{B}\right),
\end{aligned}
$$

where according to Bayes' Theorem [41] $P\left(-\sqrt{P_{t}} \leq w \leq \sqrt{P_{t}} \leq \sqrt{P_{t}} \mid c_{B}\right) \quad=\quad \frac{P\left(-\sqrt{P_{t}} \leq w \leq \sqrt{P_{t}}\right)}{P_{\mathrm{c}_{\mathrm{B}}}}$ and $P\left(w \geq+\sqrt{P_{t}} \mid c_{B}\right)=\frac{P\left(w \geq \sqrt{P_{t}}\right)}{P_{\mathrm{c}_{\mathrm{B}}}}$. Then, in terms of Lemma 1 , we are interested in finding the interval of $|w|^{2}$, and hence, (48) is given by

$$
\begin{aligned}
\mu_{\mathcal{H}_{00} B} & =E\left\{\left|y^{\prime}\right|^{2} \mid \mathcal{H}_{00_{B}}\right\} \\
& =E\left\{\left.|w|^{2}|0 \leq| w\right|^{2} \leq P_{t}\right\} \frac{P\left(-\sqrt{P_{t}} \leq w \leq \sqrt{P_{t}}\right)}{P_{\mathrm{c}_{\mathrm{B}}}} \\
& +E\left\{\left.|w|^{2}|| w\right|^{2} \geq P_{t}\right\} \frac{P\left(w \geq \sqrt{P_{t}}\right)}{P_{\mathrm{c}_{\mathrm{B}}}}
\end{aligned}
$$

Finally, according to Lemma 1, (49) takes its final shape given by (23).

\section{APPENDIX B}

\section{DERIVATION OF THE VARIANCE $V_{\mathcal{H}_{00_{B}}}$}

The derivation of the variance $V_{\mathcal{H}_{00} B}$ is based on the law of the total variance [40] and can be written as follows:

$$
\begin{aligned}
& V\left\{\left|y^{\prime}\right|^{2} \mid \mathcal{H}_{00_{B}}\right\}=V\left\{\left|y^{\prime}\right|^{2} \mid s=+\sqrt{P_{s}}, \hat{s}=+\sqrt{P_{s}}\right\} \\
& \times P\left(\hat{s}=+\sqrt{P_{s}}\right) P\left(\hat{s}=+\sqrt{P_{s}} \mid c_{B}\right) \\
& +V\left\{\left|y^{\prime}\right|^{2} \mid s=-\sqrt{P_{s}}, \hat{s}=-\sqrt{P_{s}}\right\} \\
& \times P\left(\hat{s}=-\sqrt{P_{s}}\right) P\left(\hat{s}=-\sqrt{P_{s}} \mid c_{B}\right) \\
& +E\left\{\left|y^{\prime}\right|^{2} \mid s=\sqrt{P_{s}}, \hat{s}=\sqrt{P_{s}}\right\}^{2} \\
& \times\left(1-P\left(s=\sqrt{P_{s}}\right) P\left(\hat{s}=\sqrt{P_{s}} \mid c_{B}\right)\right) \\
& \times P\left(\hat{s}=\sqrt{P_{s}}\right) P\left(\hat{s}=\sqrt{P_{s}} \mid c_{B}\right) \\
& +E\left\{\left|y^{\prime}\right|^{2} \mid s=-\sqrt{P_{s}}, \hat{s}=-\sqrt{P_{s}}\right\}^{2} \\
& \times\left(1-P\left(s=-\sqrt{P_{s}}\right) P\left(\hat{s}=-\sqrt{P_{s}} \mid c_{B}\right)\right) \\
& \times P\left(\hat{s}=-\sqrt{P_{s}}\right) P\left(\hat{s}=-\sqrt{P_{s}} \mid c_{B}\right) \\
& -2 E\left\{\left|y^{\prime}\right|^{2} \mid s=\sqrt{P_{s}}, \hat{s}=\sqrt{P_{s}}\right\} \\
& \times P\left(s=\sqrt{P_{s}}\right) P\left(s=\sqrt{P_{s}} \mid c_{B}\right)
\end{aligned}
$$

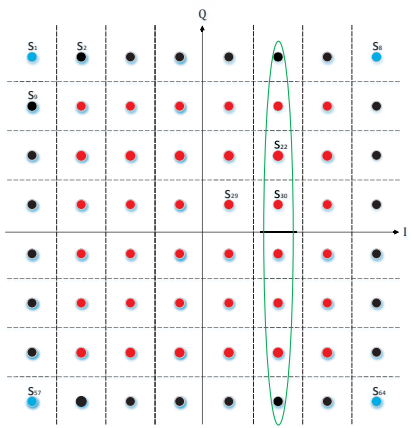

Fig. 14: Constellation for 64-QAM modulation.

$$
\begin{aligned}
& \times E\left\{\left|y^{\prime}\right|^{2} \mid s=-\sqrt{P_{s}}, \hat{s}=-\sqrt{P_{s}}\right\} \\
& \times P\left(s=-\sqrt{P_{s}}\right) P\left(s=-\sqrt{P_{s}} \mid c_{B}\right) .
\end{aligned}
$$

Then, following the same methodology as the one of the derivation of the mean $\mu_{\mathcal{H}_{00}{ }_{B}}$, the expression of (50) is simplified to (24).

\section{APPENDIX C}

\section{DERIVATION OF THE MEAN $\mu_{\mathcal{H}_{0, k=t, t=k}}$ FOR M-QAM SIGNALS}

The derivation of $\mu_{\mathcal{H}_{0, k=t, t=k}}$ under any M-QAM scenario is obtained considering the example of 64-QAM modulation. The constellation of 64-QAM is depicted in Figure 14, where the red symbols represent the inner-constellation symbols, the black symbols represent the outer-constellation symbols, while the blue symbols correspond to the outermost-constellation symbols.

- For the inner-constellation symbols, the received signal is decoded correctly according to the following constraints $C_{1}, C_{2}$ :

$$
\begin{aligned}
& C_{1}:-\sqrt{P_{t}} \leq \mathcal{R}\{w\} \leq \sqrt{P_{t}}, \\
& C_{2}:-\sqrt{P_{t}} \leq \mathcal{I}\{w\} \leq \sqrt{P_{t}} .
\end{aligned}
$$

- For the outer-constellation symbols the received signal is decoded correctly according to the following constraints $C_{1}, C_{2}$ :

$$
\begin{aligned}
& C_{1}:-\sqrt{P_{t}} \leq \mathcal{R}\{w\} \leq+\sqrt{P_{t}} \\
& C_{2}: \quad \mathcal{I}\{w\} \geq-\sqrt{P_{t}} \\
& C_{1}:-\sqrt{P_{t}} \leq \mathcal{R}\{w\} \leq+\sqrt{P_{t}} \\
& C_{2}: \quad \mathcal{I}\{w\} \leq+\sqrt{P_{t}} \\
& C_{1}: \quad \mathcal{R}\{w\} \geq-\sqrt{P_{t}} \\
& C_{2}:-\sqrt{P_{t}} \leq \mathcal{I}\{w\} \leq+\sqrt{P_{t}} \\
& C_{1}: \quad \mathcal{R}\{w\} \leq+\sqrt{P_{t}} \\
& C_{2}:-\sqrt{P_{t}} \leq \mathcal{I}\{w\}<+\sqrt{P_{t}}
\end{aligned}
$$

- For the outermost-constellation symbols the received signal is decoded correctly according to the following constraints $C_{1}, C_{2}$ :

$$
\begin{aligned}
& C_{1}: \mathcal{R}\{w\} \geq-\sqrt{P_{t}} \\
& C_{2}: \mathcal{I}\{w\} \geq-\sqrt{P_{t}}
\end{aligned}
$$

right up, 


$$
\begin{array}{ll}
C_{1}: \mathcal{R}\{w\} \geq-\sqrt{P_{t}} & \text { right down, } \\
C_{2}: \mathcal{I}\{w\} \leq+\sqrt{P_{t}} & \\
C_{1}: \mathcal{R}\{w\} \leq+\sqrt{P_{t}} & \text { left up, } \\
C_{2}: \mathcal{I}\{w\} \geq-\sqrt{P_{t}} & \\
C_{1}: \mathcal{R}\{w\} \leq+\sqrt{P_{t}} & \text { left down. } \\
C_{2}: \mathcal{I}\{w\} \leq+\sqrt{P_{t}} &
\end{array}
$$

We can generalize by saying that the intervals of interest for any M-QAM modulation scheme, under the case of correct decoding, are the following for the real part: i) $\mathcal{R}\{w\} \leq-\sqrt{P_{t}}$, ii) $-\sqrt{P_{t}} \leq \mathcal{R}\{w\} \leq \sqrt{P_{t}}$ and iii) $\mathcal{R}\{w\} \geq \sqrt{P_{t}}$. The same intervals are valid for the imaginary part. Then, the mean value for any M-QAM modulation scheme can be expressed as follows:

$$
\begin{aligned}
E\left\{\left|y^{\prime}\right|^{2} \mid \mathcal{H}_{0, k=t, t=k}\right\} & =\sum_{k=1}^{M} E\left\{\left|y^{\prime}\right|^{2} \mid s=s_{k}, \hat{s}=s_{k}\right\} \\
& \times P\left(\hat{s}=s_{k} \mid c_{M-Q}\right) P\left(s=s_{k}\right),
\end{aligned}
$$

where $c_{M-Q}$ represents the correct decision for M-QAM. Based on the constraints of (51)-(53) and following the same methodology as in Appendix A, (54) can be further determined by (55) at the top of the next page, where the factor 2 expresses the symmetry between the real and imaginary part, $P_{\text {int }}=\frac{P\left(-\sqrt{P_{t}} \leq \mathcal{R}\{w\} \leq \sqrt{P_{t}}\right) P\left(-\sqrt{P_{t}} \leq \mathcal{I}\{w\} \leq \sqrt{P_{t}}\right)}{P_{c_{M}-Q}}$, $P_{\text {ext } 1}=\frac{P\left(-\sqrt{P_{t}} \leq \mathcal{R}\{w\} \leq \sqrt{P_{t}}\right) P\left(\mathcal{I}\{w\} \geq \sqrt{P_{t}}\right)}{P_{c_{M}-Q}}$, and $P_{\text {ext } 2}=$ $\frac{P\left(\mathcal{R}\{w\} \geq \sqrt{P_{t}}\right) P\left(\mathcal{I}\{w\} \geq \sqrt{P_{t}}\right)}{P_{c_{M-}} Q}$. The first item of (55), defined as $A$, is valid for all the transmitted symbols of (54), the second and third item, defined as $B$ and $C$, respectively, are valid for all the symbols except the inter-constellation symbols and for this reason are weighted by the factor $\frac{2}{\sqrt{M}}$, while the fourth item, defined as $D$, is only valid for the outermost-constellation symbols and that's why is weighted by the factor $\frac{4}{M}$. Based on this analysis, the proof of (35) is obtained.

\section{APPENDIX D}

\section{DERIVATION OF THE MEAN $\mu_{\mathcal{H}_{0, k \neq t, t \neq k}}$ FOR M-QAM SIGNALS}

Let's assume that the symbol of interest is $s_{29}=\sqrt{P_{s}}+$ $j \sqrt{P_{s}}$. Then, the received signal is wrongly decoded according to the following constraints for the real part of noise:

$$
\begin{aligned}
C_{1}: \quad-6 \sqrt{P_{t}}+\mathcal{R}\{w\} \geq-\sqrt{P_{t}} \\
C_{2}:-\sqrt{P_{t}} \leq-4 \sqrt{P_{t}}+\mathcal{R}\{w\} \leq+\sqrt{P_{t}} \\
C_{3}:-\sqrt{P_{t}} \leq-2 \sqrt{P_{t}}+\mathcal{R}\{w\} \leq+\sqrt{P_{t}} \\
C_{4}:-\sqrt{P_{t}} \leq+2 \sqrt{P_{t}}+\mathcal{R}\{w\} \leq+\sqrt{P_{t}} \\
C_{5}:-\sqrt{P_{t}} \leq+4 \sqrt{P_{t}}+\mathcal{R}\{w\} \leq+\sqrt{P_{t}} \\
C_{6}:-\sqrt{P_{t} \leq}+6 \sqrt{P_{t}}+\mathcal{R}\{w\} \leq+\sqrt{P_{t}} \\
C_{7}: \quad+8 \sqrt{P_{t}}+\mathcal{R}\{w\} \leq+\sqrt{P_{t} .}
\end{aligned}
$$

First, we show why the constraint $C_{3}$ takes this shape and then, the rest constraints are proved similarly. The constraint $C_{3}$ is valid when we decide wrongly that the transmitted symbol is the symbol $s_{30}$ or any another symbol in the same column with $s_{30}$ (Figure 14). Thus, the condition which guarantees that the estimated symbol is $\hat{s}=s_{30}=3 \sqrt{P_{s}}+j \sqrt{P_{s}}$ is given as follows:

$$
\begin{gathered}
2 \sqrt{P_{t}} \leq \sqrt{P_{t}}+\mathcal{R}\{w\} \leq 4 \sqrt{P_{t}}=> \\
\sqrt{P_{t}} \leq+\mathcal{R}\{w\} \leq 3 \sqrt{P_{t}} .
\end{gathered}
$$

Following the steps of the proposed algorithm in Section II, it can be shown that

$$
\begin{aligned}
\mathcal{R}\left(y^{\prime}\right) & =\mathcal{R}\left\{s_{29}\right\}+\mathcal{R}\{w\}-\mathcal{R}\left\{s_{30}\right\} \\
& =\sqrt{P_{t}}+\mathcal{R}\{w\}-3 \sqrt{P_{t}}=-2 \sqrt{P_{t}}+\mathcal{R}\{w\} .
\end{aligned}
$$

Because we apply the ED in the signal of (58), we are interested in the constraints of this quantity. Therefore, adding the factor $-2 \sqrt{P_{t}}$ in (57), the constraint $C_{3}$ is proved.

Our goal is to derive the total mean of the symbols which follow the constraint $C_{1}$, the total mean of the symbols which follow the constraint $C_{2}$ and etc. For example, the constraint $C_{3}$ is validated when the transmitted symbol is $s_{29}$ and the decoded symbol is one of them in the green circle of Figure 14 , or when the transmitted symbol is $s_{30}$ and the decoded symbol is one of them in the same column with $s_{31}$ and etc. Now, for the case that the transmitted symbol is $s_{29}$ and the decoded symbol is $\hat{s}_{30}$, we can write that

$E\left\{\mathcal{R}^{2}\left\{y^{\prime}{ }_{29,30}\right\}\right\}=$

$=\frac{1}{M} E\left\{\mathcal{R}\left\{\left|y_{29,30}^{\prime}\right|^{2}\right\} \mid \mathcal{R}\{s\}=\mathcal{R}\left\{s_{29}\right\}, \mathcal{R}\{\hat{s}\}=\mathcal{R}\left\{s_{30}\right\}\right\}$

$\times P\left(\mathcal{R}\{\hat{s}\}=\mathcal{R}\left\{s_{30}\right\} \mid w_{M-Q}\right)$,

where $w_{M-Q}$ represents the wrong symbol decision for $\mathbf{M}$ QAM, while $y_{29,30}^{\prime}$ denotes the remaining signal when the transmitted symbol is $s_{29}$ and the decoded symbol is $\hat{s}_{30}$.

However, (59) can be further expressed as follows:

$$
\begin{aligned}
& E\left\{\mathcal{R}^{2}\left\{y^{\prime}{ }_{29,30}\right\}\right\}=\frac{1}{M} E\{\mathcal{E}\} \\
& \times \frac{P\left(\sqrt{P_{t}} \leq \mathcal{R}\{w\} \leq 3 \sqrt{P_{t}}\right) P\left(-\sqrt{P_{t}} \leq \mathcal{I}\{w\} \leq \sqrt{P_{t}}\right)}{P_{w_{M-Q}}} .
\end{aligned}
$$

where

$\mathcal{E}=\left|-2 \sqrt{P_{t}}+\mathcal{R}\{w\}\right|^{2} \mid-\sqrt{P_{t}} \leq-2 \sqrt{P_{t}}+\mathcal{R}\{w\} \leq \sqrt{P_{t}}$.

Furthermore, if the transmitted symbol is $s_{29}$ and the decoded symbol is $s_{20}$, the final result is

$$
\begin{aligned}
& E\left\{\mathcal{R}^{2}\left\{y^{\prime}{ }_{29,30}\right\}\right\}=\frac{1}{M} E\{\mathcal{E}\} \\
& \times \frac{P\left(\sqrt{P_{t}} \leq \mathcal{R}\{w\} \leq 3 \sqrt{P_{t}}\right) P\left(\sqrt{P_{t}} \leq \mathcal{I}\{w\} \leq 3 \sqrt{P_{t}}\right)}{P_{w_{M-Q}}} .
\end{aligned}
$$

Taking into account all these symbols, the final expression is given by $\left(s_{29}\right.$ and $\left.\hat{s}_{14}, \hat{s}_{22}, \hat{s}_{30}, \hat{s}_{38}, \hat{s}_{46}, \hat{s}_{54}, \hat{s}_{62}\right)$ :

$$
\begin{aligned}
E\left\{\mathcal{R}^{2}\left\{y^{\prime}{ }_{-2,29, C_{3}}\right\}\right\} & =\frac{1}{M} E\{\mathcal{E}\} \\
& \times \frac{P\left(\sqrt{P_{t}} \leq \mathcal{R}\{w\} \leq 3 \sqrt{P_{t}}\right)}{P_{w_{-} M-Q}},
\end{aligned}
$$

where $E\left\{\mathcal{R}^{2}\left\{y^{\prime}{ }_{-2,29, C_{3}}\right\}\right\}$ denotes the mean when the 


$$
\begin{aligned}
& E\left\{\left|y^{\prime}\right|^{2} \mid \mathcal{H}_{0, k=t, t=k}\right\}=\underbrace{2 E\left\{\mathcal{R}^{2}\left\{y^{\prime}\right\} \mid-\sqrt{P_{t}} \leq \mathcal{R}\{w\} \leq \sqrt{P_{t}}\right\} P_{\text {int }}}_{A} \\
& +\underbrace{2 E\left\{\mathcal{R}^{2}\left\{y^{\prime}\right\} \mid-\sqrt{P_{t}} \leq \mathcal{R}\{w\} \leq \sqrt{\left.P_{t}\right\} P_{\text {ext } 1}}\right.}_{B}+\underbrace{2 \frac{2}{\sqrt{M}} E\left\{\mathcal{R}^{2}\left\{y^{\prime}\right\} \mid \mathcal{R}\{w\} \geq \sqrt{P_{t}}\right\} P_{\text {ext } 2}}_{C} \\
& +\underbrace{2 \frac{4}{\sqrt{M}} E\left\{\mathcal{R}^{2}\left\{y^{\prime}\right\} \mid \mathcal{R}\{w\} \geq \sqrt{P_{t}}\right\} P_{\text {ext } 3}}_{D},
\end{aligned}
$$

transmitted symbol is $s_{29}$ and the decoded symbol has taken into account all the symbols which satisfy the constraint $C_{3}$, while the index -2 denotes the difference between the real part of the transmitted symbol with the real part of the decoded symbols, namely $\frac{\mathcal{R}_{s_{29}}-\mathcal{R}_{s_{30}}}{\sqrt{P_{t}}}$.

We assume, now, that the transmitted symbol is $s_{21}$ and the decoded symbol is one of the following: $\hat{s}_{14}, \hat{s}_{22}, \hat{s}_{30}, \hat{s}_{38}, \hat{s}_{46}, \hat{s}_{54}, \hat{s}_{62}$. Then, the mean is like in (62), namely $E\left\{\mathcal{R}^{2}\left\{y^{\prime}{ }_{-2,21, C_{1}}\right\}\right\}=E\left\{\mathcal{R}^{2}\left\{y^{\prime}{ }_{-2,29, C_{1}}\right\}\right\}$. Therefore, the total mean of the constraint $C_{3}\left(\mu_{-2}\right)$ is given by

$$
\begin{aligned}
\mu_{-2}= & =1-\frac{2}{\sqrt{M}} E\{\mathcal{E}\} \\
& \times \frac{P\left(\sqrt{P_{t}} \leq \mathcal{R}\{w\} \leq 3 \sqrt{P_{t}}\right)}{P_{w_{-} M-Q}},
\end{aligned}
$$

where we explained earlier what is the meaning of the index -2 , while the weight $\left(1-\frac{2}{\sqrt{M}}\right)$ comes from the fact that the desired symbols, based on the constraint $C_{3}$, are all the symbols, except them of the first and last column of the MQAM constellation. This analysis proves (39) for $r=-1$. Similar methodology is followed for the derivation of (40), (41), (42) and (43).

\section{REFERENCES}

[1] J. Mitola III, G. Q. Maguire, Jr., "Cognitive radios: making software radio more personal," IEEE Pers. Commun., vol. 6, no. 4, pp. 1318, Aug. 1999.

[2] S. Haykin,"Cognitive radio: brain-empowered wireless communications," J. Sel. Areas Commun., vol. 23, no. 2, pp. 201-220, Feb. 2005

[3] I. F. Akyildiz, W.-Y. Lee, M. C. Vuran, S. Mohanty, "A survey on spectrum management in cognitive radio networks," IEEE Commun. Mag., vol. 46, no. 4 , pp. 4048, Apr. 2008.

[4] M. T. Masonta, M. Mzyece, N. Ntlatlapa "Spectrum decision in cognitive radio networks: A survey," IEEE Commun. Surveys and Tutorials, vol. 15, no. 3, pp 10881107, 2013.

[5] S.-K. Sharma, T.-E. Bogale S. Chatzinotas, B. Ottersten, L.-B. Le, X. Wang, "Cognitive Radio Techniques under Practical Imperfections: A Survey," IEEE Commun. Surveys and Tutorials, vol. 17, no. 4, pp. 1858-1884, Nov. 2015.

[6] D.Cabric, S. M. Mishra, R. W. Brodersen, "Implementation issues in spectrum sensing for cognitive radios," Proc. Asilomar Comf. Signals, Syst., Comput., Pacific Grove, CA, USA, pp. 772-776, Nov. 2004.

[7] H. Urkowitz, "Energy detection of unknown deterministic signals," Proc. of the IEEE, vol. 55 , no. 4 , pp. 523-531, Apr. 1967

[8] F. Digham, M.-S. Alouini, M.K. Simon, "On the energy detection of unknown signals over fading channels," IEEE Trans. Commun., vol. 55, no. 1, pp. 21-24, Jan. 2007.

[9] R. Tandra, A. Sahai, "Fundamental limits on detection in low SNR under noise uncertainty," Proc. IEEE Int. Conf. Wireless Netw., Commun. Mobile Comput., Maui, HI, USA, vol. 1, pp, 464-469, Jun. 2005.

[10] S. Atapattu, C. Tellambura, H. Jiang, "Performance of an energy detector over channels with both multipath fading and shadowing," IEEE Trans. Wireless Commun., vol. 9, no. 12, pp. 3662-3670, Dec. 2010.

[11] P.D. Sutton, K.E. Nolan, L. E. Doyle, "Cyclostationary signatures in practical cognitive radio applications," J. Sel. Areas Commun., vol. 26, no. 1, pp. 13-24, Jan. 2008.
[12] R. Zhang, T. J. Lim, Y.-C. Liang, Y. Zeng, "Multi-Antenna Based Spectrum Sensing for Cognitive Radios: A GLRT Approach," IEEE transactions on communications, vol. 58, no. 1, Jan. 2010.

[13] P. Wang, J. Fang, N. Han, H. Li, "Multiantenna-Assisted Spectrum Sensing for Cognitive Radio," IEEE Trans. on Vehicular Technology, vol. 59, no. 4, May 2010

[14] C. Tsinos, K. Berberidis, "Decentralized Adaptive Eigenvalue-Based Spectrum Sensing for Multiantenna Cognitive Radio Systems," IEEE Trans. Wireless Commun., vol. 14, no. 3, Mar. 2015

[15] Y. C. Liang, Y. Zeng, E. Peh, A. T. Hoang, "Sensing-throughput tradeoff for cognitive radio networks," IEEE Trans. Wireless Commun., vol. 7, no. 4, pp. 13261337, Apr. 2008

[16] S. H. Song, K. Hamdi, K. B. Letaief, "Spectrum sensing with active cognitive systems,” IEEE Pers. Commun., vol. 9, no. 6, pp. 1849-1854, Jun. 2010.

[17] T. Yucek, H. Arslan, "A survey of spectrum sensing algorithms for cognitive radio applications," IEEE Commun. Surveys Tutor, vol. 11, no. 1, pp. 116-130, 2009.

[18] E. Tsakalaki, O. N. Alrabadi, A. Tatomirescu, E. Cavalho, and G. F. Pedersen, "Concurrent communication and sensing in cognitive radio devices: challenges and an enabling solution," IEEE Trans. Antennas Propag., vol. PP, no. 99, Aug. 2013.

[19] J.Heo, H. Ju,S. Park, E. Kim, D. Hong, "Simultaneous Sensing and Transmission in Cognitive Radio," IEEE Trans. Wireless Commun., vol. 13, no. 4, Apr. 2014.

[20] Y. Liao, T. Wang, L. Song, Z. Han, "Listen-and-Talk: Protocol Design and Analysis for Full-duplex Cognitive Radio Networks," IEEE Trans. on Vehicular Technology. accepted for publication, 2016.

[21] S. K. Sharma, T. E. Bogale, L. B. Le, S. Chatzinotas, X. Wang, B. Ottersten, "TwoPhase Concurrent Sensing and Transmission Scheme for Full Duplex Cognitive Radio," in Proc. IEEE VTC Spring, Sept. 2016.

[22] W. Lee, D.-H. Cho, "Concurrent spectrum sensing and data transmission scheme in a CR system," Proc. 2012 IEEE Wireless Commun. Netw. Conf., Paris, France, pp. 1326-1330., Apr. 2012.

[23] S. Stotas, A. Nallanathan, "On the throughput and spectrum sensing enhancement of opportunistic spectrum access cognitive radio networks," IEEE Trans. Wireless Comm., vol. 11, pp. 97-101, Jan. 2012.

[24] J. G. Proakis, M. Salehi, Digital Communications, 5th Edition, McGraw-Hill, 2008

25] G. P. Wadsworth, J. G. Bryan, Introduction to Probability and Random Variables, McGraw-Hill, page 52, 1960.

[26] N.L. Johnson, S. Kotz, N. Balakrishnan, Continuous Univariate Distributions, Volume 1, Wiley, 1994

[27] P. Damien, G. S. Walker, "Sampling truncated normal, beta, and gamma densities," Journal of Computational and Graphical Statistics, 2001.

[28] J. Rice, Mathematical Statistics and Data Analysis, Second Edition, Duxbury Press, 1995.

[29] P. Billingsley, Probability and Measure, Third Edition, John Wiley and Sons, 1995, page 357.

[30] C. S. Coffey, K. E. Muller, "Properties of Doubly-Truncated Gamma Variables," Commun. Stat. Theory Methods, Feb. 2000.

[31] E. Marchand, "Computing the moments of a truncated noncentral chi-square distribution," Journal of Statistical Computation and Simulation, Mar. 2007.

[32] P. J. Dhrymes, "Moments of truncated (normal) distributions", 2005.

[33] D. Tse, P. Viswanath, Fundamentals of Wireless Communication, Cambridge University Press, 2005.

[34] A. Taherpour, M. Nasiri-Kenari, S. Gazor, "Multiple antenna Spectrum Sensing in Cognitive Radios," IEEE Trans. Wireless Commun., vol. 9, no. 2, Feb. 2010

[35] D. Cabric, A. Tkachenko and R. W. Brodersen, "Experimental study of spectrum sensing based on energy detection and network cooperation," First international workshop on Technology and policy for accessing spectrum, 2006.

[36] D. Cabric, A. Tkachenko and R. W. Brodersen, "Spectrum Sensing Measurements of Pilot, Energy, and Collaborative Detection," IEEE Military Communication Conference, 2006. MILCOM 2006., pp.1,7, 23-25 Oct. 2006.

[37] R. Deng, J. Chen, C. Yuen, P. Cheng and Y. Sun, "Energy-Efficient Cooperative Spectrum Sensing by Optimal Scheduling in Sensor-Aided Cognitive Radio Networks," IEEE Transactions on Vehicular Technology, vol.61, no.2, pp.716,725, Feb 2012.

[38] C. Politis, S. Maleki, C. Tsinos, S. Chatzinotas, B. Ottersten, "On-board the satellite interference detection with imperfect signal cancellation," IEEE Intern. Workshop on Sig. Proc. Advanc.in Wirel. Comm., Edinburgh, Scotland, Jul. 2016.

[39] Q. Huang, P.J. Chung, "An F-Test Based Approach for Spectrum Sensing in Cognitive Radio," IEEE Trans. Wireless Commun., vol. 12, no. 8, Aug. 2013. 
[40] N. A. Weiss, A Course in Probability, Addison - Wesley, 2005, pages 385-386. [41] A. Stuart, K. Ord, (1994), Kendall's Advanced Theory of Statistics: Volume I Distribution Theory, Wiley, Jun. 1994.

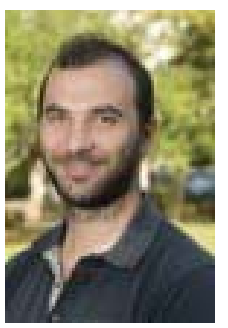

Christos Politis was born in Athens, Greece, in 1986 He received the Dipl.-Eng. degree in Electrical and Computer Engineering from the University of Patras, Greece, in 2011; the MSc degree in Space Communication Systems from ISAE-SUPAERO, France, in 2006; and a second MSc degree in Communications and Signal Processing from the University of Newcastle, UK, 2014. He is currently working toward the $\mathrm{Ph} . \mathrm{D}$. degree with the research group SIGCOM in the Interdisciplinary Centre for Security, Reliability and Trust (SnT), University of Luxembourg. His research interests includes signal processing for wireless and satellite communications with focus on interference detection.

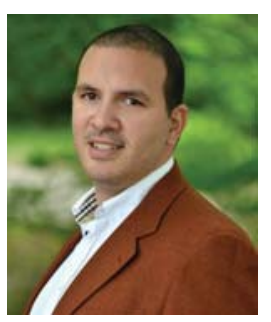

Konstantinos P. Liolis was born in Athens, Greece, in 1981. He received the Dipl.-Eng. degree in Electrical and Computer Engineering from the National Technical University of Athens (NTUA), Greece, in 2004; the MSc degree in Electrical Engineering from the University of California at San Diego (UCSD), USA, in 2006; and the $\mathrm{PhD}$ degree in Electrical Engineering from NTUA in 2011. From 2004 to 2006, he was Research Assistant at the California Institute for Telecommunications and Information Technology (Cal-IT2), San Diego, USA. From 2006 to 2008, he was Communication Systems Engineer at the European Space Agency, Research and Technology Centre (ESA/ESTEC), the Netherlands. From 2008 to 2012, he was R\&D Project Manager at Space Hellas S.A., Greece. In 2011, he also fulfilled his military service as 2nd Class Petty Officer (Specialization: Computer Network Engineer) in the Hellenic Navy. Since 2012, he has been with SES S.A., Luxembourg, where he is currently Senior Systems Engineer managing technology innovation projects. He has published more than 50 scientific papers in international peer-reviewed journals, conference proceedings and book chapters, in areas mainly related to satellite communications. He has also numerous contributions to the ETSI, DVB and ITU-R international standardization bodies, and has actively participated in more than 35 R\&D\&I projects funded by EU, ESA and National programs

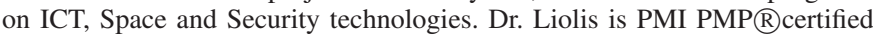
project manager and is a Member of the Technical Chamber of Greece (TEE) He has served as TPC Member for several international conferences and as Reviewer for several major international journals and conferences. He received a Best Student Paper Award in IEEE RAWCON 2006.

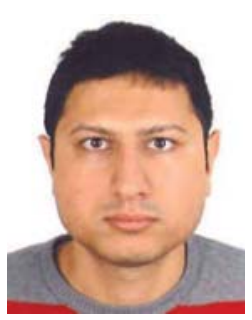

Sina Maleki (M13) received the B.Sc. degree from the Iran University of Science and Technology, Tehran, Iran, in 2006, and the M.Sc. and Ph.D. degrees from the Delft University of Technology, Delft, The Netherlands, in 2009 and 2013, respectively. From 2008 to 2009, he was an intern student with the Philips Research Center, Eindhoven, The Netherlands, researching on spectrum sensing for cognitive radio networks. Since 2013, he has been with the Interdisciplinary Centre for Security, Reliability and Trust, University of Luxembourg, where he is working on cognitive radio for satellite communications as well as interference detection and localization in satellite communications, within the EU H2020 Project SANSA, EU FP7 Project CoRaSat, as well as Luxembourgish National Projects SATSENT and SeMIGod.

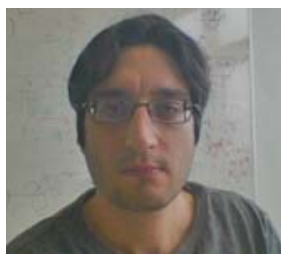

Christos G. Tsinos (S08-M14) received the Diploma degree in computer engineering and informatics, the MSc and the $\mathrm{PhD}$ degree in signal processing and communication systems and MSc in applied mathematics from the University of Patras, Greece, in 2006, 2008, 2013 and 2014 respectively. From August 2014 to June to 2015 he was a Postdoctoral Researcher at University of Patras. Since July 2015 he joined as a Research Associate the Interdisciplinary Centre for Security, Reliability and Trust (SnT), University of Luxembourg, Luxembourg. He is involved or was involved in the past in a number of different $R \& D$ projects funded by national and/or EU funds. He is currently the PI of R\&D Project ECLECTIC (Energy and CompLexity EffiCienT mIllimeter-wave Large-Array Communications), funded under FNR CORE Framework. His current research interests include signal processing for mmWave, massive MIMO, cognitive radio and satellite communications and hyperspectral image processing, as well. Dr. Tsinos is a member of the Technical Chamber of Greece.

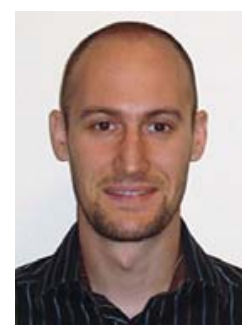

Symeon Chatzinotas (S06-M09-SM-13) is currently the Deputy Head of the SIGCOM Research Group, Interdisciplinary Centre for Security, Reliability, and Trust, University of Luxembourg, Luxembourg. In the past, he has worked on numerous R\&D projects for the Institute of Informatics Telecommunications, National Center for Scientific Research Demokritos, Institute of Telematics and Informatics, Center of Research and Technology Hellas, and Mobile Communications Research Group, Center of Communication Systems Research, University of Surrey, Surrey, U.K. He has received the M.Eng. degree in telecommunications from Aristotle University of Thessaloniki, Thessaloniki, Greece, and the M.Sc. and Ph.D. degrees in electronic engineering from the University of Surrey, Surrey, U.K., in 2003, 2006, and 2009, respectively. Dr. Chatzinotas has more than 200 publications, 1600 citations and an H-Index of 22 according to Google Scholar. He is the co-recipient of the 2014 Distinguished Contributions to Satellite Communications Award, and Satellite and Space Communications Technical Committee, IEEE Communications Society, and CROWNCOM 2015 Best Paper Award. His research interests include multiuser information theory, co-operative/cognitive communications and wireless networks optimization. 


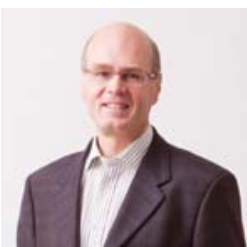

Björn Ottesten (S87-M89-SM99-F04) was born in Stockholm, Sweden, in 1961. He received the M.S. degree in electrical engineering and applied physics from Linkping University, Linkping, Sweden, in 1986, and the Ph.D. degree in electrical engineering from Stanford University, Stanford, CA, USA, in 1989. He has held research positions at the Department of Electrical Engineering, Linkping University, the Information Systems Laboratory, Stanford University, the Katholieke Universiteit Leuven, Leuven, Belgium, and the University of Luxembourg, Luxembourg.

From 1996 to 1997, he was the Director of Research at ArrayComm Inc, a start-up in San Jose, CA, based on his patented technology. In 1991, he was appointed a Professor of Signal Processing with the Royal Institute of Technology (KTH), Stockholm, Sweden. From 1992 to 2004, he was the Head of the Department for Signals, Sensors, and Systems, KTH, and from 2004 to 2008, he was the Dean of the School of Electrical Engineering, KTH. Currently, he is the Director for the Interdisciplinary Centre for Security, Reliability and Trust, University of Luxembourg. As Digital Champion of Luxembourg, he acts as an Adviser to the European Commission. Dr. Ottersten has served as Associate Editor for the IEEE TRANSACTIONS ON SIGNAL PROCESSING and on the Editorial Board of IEEE Signal Processing Magazine. He is currently editor in chief of EURASIP Signal Processing Journal and a member of the editorial boards of EURASIP Journal of Advances Signal Processing and Foundations and Trends of Signal Processing. He has coauthored journal papers that received the IEEE Signal Processing Society Best Paper Award in 1993, 2001, 2006, and 2013 and three IEEE conference papers receiving Best Paper Awards. Dr. Ottersten is a Fellow of the IEEE and EURASIP. In 2011 he received the IEEE Signal Processing Society Technical Achievement Award. He has received the European Research Council advanced research grant twice, 2009-2013 and 2017-2021. 